\title{
Minimum distance estimation of parametric Lorenz curves based on grouped data
}

\author{
Gholamreza Hajargasht \\ Swinburne Business School \\ William E. Griffiths \\ University of Melbourne
}

\begin{abstract}
The Lorenz curve, introduced more than 100 years ago, remains as one of the main tools for analysis of inequality. International institutions such as the World Bank collect and publish grouped income data in the form of population and income shares for a large number of countries. These data are often used for estimation of parametric Lorenz curves which in turn form the basis for most inequality analyses. Despite the prevalence of parametric estimation of Lorenz curves from grouped data, and the existence of well-developed nonparametric methods, a formal description of rigorous methodology for estimating parametric Lorenz curves from grouped data is lacking. We fill this gap. Building on two data generating mechanisms, efficient methods of estimation and inference are described; several results useful for comparing the two methods of inference, and aiding computation, are derived. Simulations are used to assess the estimators, and curves are estimated for some example countries. We also show how the proposed methods improve upon World Bank methods and make recommendations for improving current practices.
\end{abstract}

Keywords: Minimum Distance, GMM, GB2 Distribution, Quantile Function Estimation

JEL Classification: C13, C16, D31

\section{Corresponding author:}

William Griffiths

Department of Economics

University of Melbourne

Vic 3010, Australia

Phone: +613 83443622

Email for Griffiths: wegrif@unimelb.edu.au

Email for Hajargasht: har@unimelb.edu.au 


\section{Introduction}

The Lorenz curve has a long history of use as a basis for welfare comparisons. Despite this history, the abundance of parametric specifications and estimators that have appeared in the literature, and the existence of well-developed distribution-free methods of analysis, much of current practice for estimating parametric Lorenz curves lacks a solid statistical foundation. The objective of this paper is to alleviate this deficiency. We describe a sound statistical framework for using grouped data to estimate parametric Lorenz curves and their by-products. We recognise that the grouped data may originate from two possible data generating processes (DGPs) - one where group proportions are predetermined, and group limits are random (DGP1), and one where group limits are fixed a priori, and group proportions are random (DGP2). Using minimum distance (MD) estimation, for each DGP we specify suitable moment conditions, derive closed forms for the optimal weight matrices, and show that, under equivalent conditions, the two MD estimators have the same asymptotic covariance matrix.

Despite the increasing availability of microlevel data in the form of single observations, there is a continuing need for sound methodology for use with grouped data. Institutions such as the World Bank and the World Institute for Development Economics Research collect and publish data in the form of population and income shares for a number of groups. ${ }^{1}$ These data are used to estimate parametric Lorenz curves which, when combined with information on mean incomes, form the basis for poverty and inequality analyses. Large scale regional and global studies of inequality and poverty, involving many countries over several time periods, use grouped data to track changes in inequality and make cross-country comparisons. ${ }^{2}$

\footnotetext{
${ }^{1}$ Historically, the typical number of groups has been between 10 and 20. More recently, the World Bank has published 100 groups for some countries in some years. However, in many instances, data availability is still restricted to the smaller numbers of groups.

2 Examples of large-scale studies which use grouped data are Anand and Segal (2008), Bourguignon and Morrisson (2002), Chen and Ravallion (2011), Chotikapanich et al. (2012), Jorda and Ni ñ o-Zarazúa (2016), Pinkovskiy and Sala-i-Martin (2014) and Sala-i-Martin (2006).
} 
For circumstances in which micro-level data are available, there is a rich literature describing nonparametric methods of estimation. ${ }^{3}$ Relative to nonparametric methods, our proposed parametric methodology has both advantages and disadvantages. Its obvious disadvantage is the possibility of misspecification of either the income distribution or the Lorenz curve, and the implications of this misspecification for properties of estimators. If this disadvantage can be mitigated through choice of a "good" or flexible parametric specification, then the advantages of a parametric specification are greater precision of estimation, particularly in the tails of the distribution, and the ability to draw inferences about Lorenz ordinates at points in between observed data points. Also, when using grouped data, a parametric specification avoids the nonparametric requirement for data on group limits and group variances, a requirement often not met in published data.

Sub-optimal methods for estimating parametric Lorenz curves are common in the literature. Typically, they involve linear or nonlinear least squares, or a form of generalized least squares, applied directly to income and population shares, or functions of them. ${ }^{4}$ The World Bank has used least squares to estimate both the general quadratic and the beta Lorenz curves, with the better-fitting one being chosen for later analysis. ${ }^{5}$ While naïve application of least squares may be a reasonable "fitting device", it does not recognise that observations on cumulative proportions are correlated and heteroskedastic. Attempts to mitigate these concerns, e.g., Kakwani and Podder (1976), Sarabia et al. (1999) and Chotikapanich and Griffiths (2002), do not come to grips with the need to specify a suitable underlying data generation process.

\footnotetext{
${ }^{3}$ See, for example, Gastwirth (1972), Goldie (1977), Beach and Davidson (1983), Gastwirth and Gail (1985), Bishop et al (1989), Cowell and Victoria-Feser (2002), the review by Maasoumi (1999), and references cited therein.

${ }^{4}$ Some examples are Kakwani and Podder (1976), Basmann et al (1990), Chotikapanich (1993) and Wang et al. (2011). Further examples and references can be found in the edited book, Chotikapanich (2008).

${ }^{5}$ Datt (1998) describes the World Bank's methods. An example of where the estimates are used for poverty and inequality analysis is Chen and Ravallion (2010).
} 
We propose MD estimators for each method of grouping. For DGP2 (random group proportions) our description of the estimator is brief. It is an extension of that in Hajargasht et al. (2012), modified to accommodate cumulative proportions convenient for Lorenz curve specifications. More attention is paid to DGP1 (random group limits). This method of grouping is the more common one for summarising income distribution data, and many of the suboptimal estimators suggested in the literature can be considered within a subset of this framework. A particularly pleasing characteristic of the subset framework is that Lorenz curve parameters, or those of a corresponding income distribution, can be estimated without knowledge of group limits which are frequently unobserved. ${ }^{6}$

In Section 2 we introduce the notation and describe the two data generating processes. Sections 3 and 4 are devoted to estimation under DGP1 and DGP2, respectively. In Section 3 we highlight how traditional methods can be viewed as sub-optimal special cases of the MD framework. The relationship between the estimators for each of the two DGPs is examined in Section 5. We show how the two estimators have the same asymptotic covariance matrix if the groupings are a priori consistent. We also indicate how results for the weight matrix for the DGP2 estimator can facilitate computations for the DGP1 estimator. In Section 6 we summarise the results from some Monte Carlo examples; the complete Monte Carlo results are given in a supplementary appendix. Lorenz curves are estimated for some example countries in Section 7; we show how the World Bank's estimates can be improved by using alternative estimation methods and/or alternative Lorenz curve specifications.

\section{Definitions and two data generating processes}

Let $y$ denote income, and let $f(y)$ and $F(y)$ be its density and cumulative distribution functions, respectively. We treat the support of $y$ as $[0, \infty)$, but our results hold for a finite

\footnotetext{
${ }^{6}$ With DGP2, unknown group limits are treated as unknown parameters and estimated. In the subset framework of DGP1, they are not required.
} 
support $\left[y_{\min ,} y_{\max }\right]$ providing $f(y)$ is nonzero within this interval. Let $\mu=\int_{0}^{\infty} y f(y) d y$ be the mean of the distribution. The Lorenz curve (LC) relating the cumulative proportion of income $L$ to the cumulative proportion of population $c$ is given by

$$
L(c)=\frac{1}{\mu} \int_{0}^{c} F^{-1}(t) d t \quad 0 \leq c \leq 1
$$

The generalized Lorenz curve (GLC) which can be used for making welfare comparisons based on both the level of income and the degree of inequality is given by

$$
G(c)=\mu L(c)=\int_{0}^{c} F^{-1}(t) d t=\mu F_{1}\left(F^{-1}(c)\right) \quad 0 \leq c \leq 1
$$

$F_{1}(y)=(1 / \mu) \int_{0}^{y} t f(t) d t$ is the first moment distribution function. We focus on estimation of the GLC; in Appendix D we show how estimation can be modified to cover the standard LC.

We consider estimation of a vector of parameters $\phi$ which can be the parameters of a parametric GLC specified directly, or the parameters of a parametric income distribution from which a GLC has been derived. Any non-decreasing convex function $G(c)$ where $c \in[0,1]$, $G\left(0^{+}\right)=0$, and $G\left(1^{-}\right)=\mu$, can be a GLC. ${ }^{7}$ An example considered later in the paper is

$$
G(c ; \phi)=\mu c^{\beta_{1}}\left\{1-(1-c)^{\beta_{2}}\right\}^{\beta_{3}}
$$

This curve was proposed by Sarabia et al. (1999) (hereafter the SCS curve). If our objective is to estimate the GLC in (2.3), then $\phi^{\prime}=\left(\beta_{1}, \beta_{2}, \beta_{3}, \mu\right)$.

To illustrate GLC estimation that originates from specification of a parametric income distribution, consider the generalized beta distribution of the second kind (GB2)

$$
f(y ; \phi)=\frac{a y^{a p-1}}{b^{a p} \mathrm{~B}(p, q)\left[1+(y / b)^{a}\right]^{p+q}}
$$

\footnotetext{
${ }^{7}$ See Sarabia (2008) and Kleiber and Kotz (2003) for reviews of alternative parametric specifications that have appeared in the literature, and Wang et al (2011) and Wang and Smyth (2015) for further proposals.
} 
with positive parameters $\phi^{\prime}=(b, p, q, a)$, and with $\mathrm{B}(p, q)$ denoting the beta function. Its cumulative distribution function (cdf) is given by

$$
F(y ; \phi)=\frac{1}{\mathrm{~B}(p, q)} \int_{0}^{u} t^{p-1}(1-t)^{q-1} d t=B(u ; p, q)
$$

where $u=y^{a} /\left(b^{a}+y^{a}\right)$, and $B(u ; p, q)$ is the cdf for the normalised beta distribution defined on $(0,1)$. Its mean is $\mu=b \mathrm{~B}(p+1 / a, q-1 / a) / \mathrm{B}(p, q)$, and its GLC can be written as ${ }^{8}$

$$
G(c ; \phi)=\mu B\left(B^{-1}(c ; p, q) ; p+1 / a, q-1 / a\right) \quad q>1 / a
$$

To introduce the two DGPs corresponding to the two methods for grouping observations, we begin by defining some notation that we later modify slightly according to the DGP. ${ }^{9}$ Assume that a sample of $T$ observations $\left(y_{1}, y_{2}, \ldots, y_{T}\right)$ is randomly drawn from the income distribution, and placed into $N$ income groups defined by the group boundaries $\left(z_{0}, z_{1}\right)$, $\left(z_{1}, z_{2}\right), \ldots,\left(z_{N-1}, z_{N}\right)$, where $z_{0}=0$ and $z_{N}=\infty$. Let $T_{i}$ be the number of observations and $M_{i}$ total income in the $i$-th group. Also, let $c_{i}=T^{-1} \sum_{j=1}^{i} T_{j}$ and $\tilde{y}_{i}=T^{-1} \sum_{j=1}^{i} M_{j}, i=1,2, \ldots, N$ be the cumulative proportion of observations and the cumulative mean income share for each group, respectively; $c_{N}=1$ and $\tilde{y}_{N}=T^{-1} \sum_{t=1}^{T} y_{t}$.

\subsection{DGP1: fixed $c_{i}$ and stochastic $z_{i}$}

Here the observations are grouped such that the proportion of observations in each group is pre-specified. Examples are 10 groups with $10 \%$ of the observations in each group or 20 groups with $5 \%$ of the observations in each group. In this case, the cumulative proportions $c_{i}$ are fixed (non-random) and the sample group boundaries as well as the average cumulative incomes $\tilde{y}_{i}$,

\footnotetext{
${ }^{8}$ For estimating (2.5), it turns out to be computationally more convenient to parameterize it as $\phi^{\prime}=(\mu, p, q, a)$.

${ }^{9}$ In an unpublished work, Wu (2006) has also distinguished between the two cases and has derived estimators for densities with grouped data. Here, our focus is on Lorenz curves and therefore the resulting moment conditions and weight matrix are different.
} 
are random variables. The sample group boundaries are given by $\tilde{z}_{i}=\max \left\{y_{t} h_{i}\left(y_{t}\right)\right\}$, where $h_{i}\left(y_{t}\right)$ is an indicator function equal to one if $y_{t}$ is in the $i$-th group and zero otherwise, and where we use a tilde “ ” on $\tilde{z}_{i}$ to recognise its randomness. Define vectors $\tilde{\mathbf{z}}^{\prime}=\left(\tilde{z}_{1}, \tilde{z}_{2}, \ldots, \tilde{z}_{N-1}\right)$ and $\tilde{\mathbf{y}}_{L}^{\prime}=\left(\tilde{y}_{L 1}, \tilde{y}_{L 2}, \ldots, \tilde{y}_{L N}\right)$. The subscript $L$ on $\tilde{\mathbf{y}}_{L}$ is used to distinguish it from the average cumulative incomes that we later introduce under DGP2. The sample group boundary $\tilde{z}_{i}$ is an estimator for the quantile $F^{-1}\left(c_{i} ; \phi\right)$; the average cumulative income $\tilde{y}_{L i}$ is an estimator for the GLC ordinate $G\left(c_{i} ; \phi\right)$. If the available grouped data includes information on $\tilde{\mathbf{z}}$ as well as data on $\mathbf{c}^{\prime}=\left(c_{1}, c_{2}, \ldots, c_{N-1}\right)$ and $\tilde{\mathbf{y}}_{L}$, then both $\tilde{\mathbf{z}}$ and $\tilde{\mathbf{y}}_{L}$ can be used to estimate $\phi$. If the sample group boundaries are not available, one can proceed with estimation based solely on $\tilde{\mathbf{y}}_{L} \cdot{ }^{10}$

\subsection{DGP2: stochastic $c_{i}$ and fixed $z_{i}$}

The second way in which grouping can take place is with pre-specified group boundaries in which case $\mathbf{z}^{\prime}=\left(z_{1}, z_{2}, \ldots, z_{N-1}\right)$ is predetermined and the cumulative population shares $c_{i}$ and average cumulative incomes $\tilde{y}_{i}$ are random. An example is $z_{1}=\$ 30,000, z_{2}=\$ 60,000$, $z_{3}=\$ 90,000$, and so on, although equal intervals are not essential. In this case we use the notation $\tilde{c}_{i}=T^{-1} \sum_{j=1}^{i} T_{j}$ to denote an estimator for $F\left(z_{i} ; \phi\right)$, with $\tilde{\mathbf{c}}^{\prime}=\left(\tilde{c}_{1}, \tilde{c}_{2}, \ldots, \tilde{c}_{N-1}\right)$. Introducing a subscript $m$ for the cumulative incomes, we have $\tilde{\mathbf{y}}_{m}^{\prime}=\left(\tilde{y}_{m 1}, \tilde{y}_{m 2}, \ldots, \tilde{y}_{m N}\right)$ where $\tilde{y}_{m i}$ is an estimator for $m\left(z_{i} ; \phi\right)=\mu F_{1}\left(z_{i} ; \phi\right)=\int_{0}^{z_{i}} t f(t) d t$. Both $\tilde{\mathbf{c}}$ and $\tilde{\mathbf{y}}_{m}$ can be used to estimate $\phi$; if the group boundaries are not provided, they can be estimated along with $\phi$.

\footnotetext{
${ }^{10}$ The possible unavailability of group boundaries is in line with the data on PovcalNet where population and income shares and mean income are provided, from which the $c_{i}$ and $\tilde{y}_{i}$ can be readily calculated; the $z_{i}$ traditionally have not been provided, but have recently been posted for some countries.
} 


\section{Estimator for DGP1, fixed $c_{i}$ and stochastic $z_{i}$}

Let $\mathbf{F}^{-1}(\mathbf{c} ; \phi)$ be an $(N-1)$ dimensional vector with $F^{-1}\left(c_{i} ; \phi\right)$ as its $i$-th element, and let $\mathbf{G}(\mathbf{c} ; \phi)$ be an $N$-dimensional vector with $G\left(c_{i} ; \phi\right)$ as its $i$-th element. To estimate $\phi$, we can use $\tilde{\mathbf{z}}$ as an estimator for $\mathbf{F}^{-1}(\mathbf{c} ; \phi)$, and $\tilde{\mathbf{y}}_{L}$ as an estimator for $\mathbf{G}(\mathbf{c} ; \phi)$. Two methods for estimating $\phi$ are considered. The first uses information on both the sample group bounds $\tilde{\mathbf{z}}$ and the average cumulative incomes $\tilde{\mathbf{y}}_{L}$; the second is relevant when data on $\tilde{\mathbf{z}}$ are not available - the typical scenario in the literature where only $\tilde{\mathbf{y}}_{L}$ is used. For the moment, we assume that tractable closed form expressions are available for both the quantile function $F^{-1}(c ; \phi)$ and the GLC $G(c ; \phi)$.

For a sample drawn from a population with cdf $F(y)$, where $f\left(z_{i}\right)=F^{\prime}\left(z_{i}\right)>0$, and $c_{i}=F\left(z_{i}\right)$, we have (see e.g., Beach and Davidson 1983 and references cited therein)

$$
\sqrt{T}\left(\tilde{\mathbf{z}}-\mathbf{F}^{-1}(\mathbf{c} ; \phi)\right) \rightarrow N\left(\mathbf{0}, \boldsymbol{\Omega}_{L, 11}\right)
$$

where

$$
\boldsymbol{\Omega}_{L, 11}=\left[\begin{array}{ccc}
\frac{c_{1}\left(1-c_{1}\right)}{f\left(z_{1}\right)^{2}} & \cdots & \frac{c_{1}\left(1-c_{N-1}\right)}{f\left(z_{1}\right) f\left(z_{N-1}\right)} \\
\vdots & \ddots & \vdots \\
\frac{c_{1}\left(1-c_{N-1}\right)}{f\left(z_{1}\right) f\left(z_{N-1}\right)} & \cdots & \frac{c_{N-1}\left(1-c_{N-1}\right)}{f\left(z_{N-1}\right)^{2}}
\end{array}\right]
$$

To include $\tilde{\mathbf{y}}_{L}$ in the estimation procedure, we use results from Beach and Davidson (1983) and Cowell and Victoria-Feser (2002) to obtain

$$
\sqrt{T}\left(\tilde{\mathbf{y}}_{L}-\mathbf{G}(\mathbf{c} ; \phi)\right) \rightarrow N\left(\mathbf{0}, \boldsymbol{\Omega}_{L, 22}\right)
$$

where the elements in $\boldsymbol{\Omega}_{L, 22}$ are

$$
\left[\boldsymbol{\Omega}_{L, 22}\right]_{i, j}=m_{i}^{(2)}+\left(c_{i} z_{i}-G\left(c_{i}\right)\right)\left(z_{j}-c_{j} z_{j}+G\left(c_{j}\right)\right)-z_{i} G\left(c_{i}\right) \quad \text { for } i \leq j
$$

with 


$$
m_{i}^{(2)}=m^{(2)}\left(z_{i}\right)=\int_{0}^{z_{i}} y^{2} f(y) d y
$$

Symmetry is used to establish the elements for $i>j$. To combine $\tilde{\mathbf{z}}$ and $\tilde{\mathbf{y}}_{L}$ in an estimation procedure, we also need the asymptotic covariance matrix $\boldsymbol{\Omega}_{L, 12}=\operatorname{cov}\left(\sqrt{T} \tilde{\mathbf{z}}, \sqrt{T} \tilde{\mathbf{y}}_{L}\right)$. In Appendix A we show that

$$
\begin{array}{ll}
{\left[\mathbf{\Omega}_{L, 12}\right]_{i, j}=\frac{c_{i}\left(G\left(c_{j}\right)-z_{j} c_{j}+z_{j}\right)-G\left(c_{i}\right)}{f\left(z_{i}\right)}} & \text { for } i \leq j \\
{\left[\mathbf{\Omega}_{L, 12}\right]_{i, j}=\frac{\left(c_{i}-1\right)\left(G\left(c_{j}\right)-z_{j} c_{j}\right)}{f\left(z_{i}\right)}} & \text { for } i \geq j
\end{array}
$$

Collecting all these results together, we have

$$
\sqrt{T}\left[\begin{array}{c}
\tilde{\mathbf{z}}-\mathbf{F}^{-1}(\mathbf{c} ; \phi) \\
\tilde{\mathbf{y}}_{L}-\mathbf{G}(\mathbf{c} ; \phi)
\end{array}\right] \rightarrow N\left(\mathbf{0}, \boldsymbol{\Omega}_{L}\right) \quad \text { where } \boldsymbol{\Omega}_{L}=\left[\begin{array}{ll}
\boldsymbol{\Omega}_{L, 11} & \boldsymbol{\Omega}_{L, 12} \\
\boldsymbol{\Omega}_{L, 12}^{\prime} & \boldsymbol{\Omega}_{L, 22}
\end{array}\right]
$$

This result does not depend on any parametric assumptions. Thus, $\tilde{y}_{L i}$ is a nonparametric estimate for the GLC ordinate $G\left(c_{i} ; \phi\right)$. If sufficient information is available to estimate $\left[\boldsymbol{\Omega}_{L, 22}\right]_{i, i}$ (see 3.4)), then this estimate can be used to assess the reliability of $\tilde{y}_{L i}$ as a nonparametric estimate for $G\left(c_{i} ; \phi\right)$. The required information is $\tilde{z}_{i}, \tilde{y}_{L i}$, and an estimate for $m_{i}^{(2)}$. While these quantities are readily available with micro-level data, for grouped data they require knowledge of group variances and group limits. With grouped data, group limits are frequently not provided and, as far as we are aware, group variances are never provided. This problem can be overcome by making a parametric assumption about either the income distribution or the GLC. Making such an assumption means that $m_{i}^{(2)}, z_{i}, f\left(z_{i}\right)$ and $G\left(c_{i}\right)$ can be expressed in terms of the parameters of the income distribution or the GLC, and a MD framework can be set up to estimate those parameters. 
Introducing a zero subscript to distinguish the underlying parameter vector $\phi_{0}$ from other possible values of $\phi$, from MD theory ${ }^{11,12}, \hat{\phi}_{L}$ defined by

$$
\hat{\phi}_{L}=\arg \min _{\phi} \mathbf{H}_{L}^{\prime}(\phi) \tilde{\mathbf{W}}_{L} \mathbf{H}_{L}(\phi)
$$

(i) is a consistent estimator of $\phi_{0}$, and

(ii) $\sqrt{T}\left(\hat{\phi}_{L}-\phi_{0}\right) \stackrel{d}{\longrightarrow} N\left(\mathbf{0}, \mathbf{V}_{L}\right) \quad$ where $\quad \mathbf{V}_{L}=\left(\frac{\partial \mathbf{H}_{L}^{\prime}}{\partial \phi} \mathbf{W}_{L} \frac{\partial \mathbf{H}_{L}}{\partial \phi^{\prime}}\right)^{-1}$

Here, $\mathbf{W}_{L}=\Omega_{L}^{-1}\left(\phi_{0}\right), \tilde{\mathbf{W}}_{L}=\Omega_{L}^{-1}(\tilde{\phi})$, where $\tilde{\phi}$ is any consistent estimator for $\phi_{0}$, and

$$
\mathbf{H}_{L}(\phi)=\left[\begin{array}{c}
\tilde{\mathbf{z}}-\mathbf{F}^{-1}(\mathbf{c} ; \phi) \\
\tilde{\mathbf{y}}_{L}-\mathbf{G}(\mathbf{c} ; \phi)
\end{array}\right]
$$

For application of this result, it is useful to distinguish between two scenarios, one where the group limits $\tilde{\mathbf{z}}$ are observable and one where they are not.

\subsection{Estimation with known $\tilde{\mathbf{z}}$}

In this case, expressions are required for the quantile function $F^{-1}\left(c_{i} ; \phi\right)$, the GLC $G\left(c_{i} ; \phi\right)$, and the weight matrix $\mathbf{W}_{L}$, in terms of the unknown parameters $\phi$. First, suppose we begin with a GLC specification such as that given in (2.3), then the following lemma can be used to derive the required quantities.

Lemma 1: If $G\left(c_{i} ; \phi\right)$ satisfies the conditions of a GLC, is twice differentiable with $\partial^{2} G\left(c_{i}, \phi\right) / \partial c_{i}^{2}>0$ and $c_{i}=F\left(z_{i} ; \phi\right)$, then the following relationships hold

$$
\text { (i) } \quad Z_{i}=F^{-1}\left(c_{i} ; \phi\right)=\frac{\partial G\left(c_{i} ; \phi\right)}{\partial c_{i}}
$$

\footnotetext{
${ }^{11}$ See Newey and McFadden (2004), Hansen (1982), and Ferguson (1958) for general information about minimum distance estimators, and regularity conditions.

12 Note that there are other parametric estimators for use with grouped data. An example with excellent robustness properties is the minimum power divergence estimator (MPE) of Cressie and Read (1984). See Victoria-Feser and Ronchetti (1997) and Victoria-Feser (2000) for applications to income distributions.
} 


$$
\begin{aligned}
& \text { (ii) } f\left(z_{i} ; \phi\right)=\frac{1}{\partial^{2} G\left(c_{i} ; \phi\right) / \partial c_{i}^{2}} \\
& \text { (iii) } m_{i}^{(2)}\left(z_{i} ; \phi\right)=\int_{0}^{z_{i}} y^{2} f(y ; \phi) d y=\int_{0}^{c_{i}}\left(\frac{\partial G\left(x_{i} ; \phi\right)}{\partial x_{i}}\right)^{2} d x_{i}
\end{aligned}
$$

Proof: (i) is a well-known result in the Lorenz curve literature (see e.g. Gastwirth 1971), (ii) is also well-known and can obtained by using (i) and applying the chain rule, and (iii) is obtained by using (i) and a change of variable for integration.

As an example, applying (i) to the Lorenz curve in (2.3), yields

$$
z_{i}=F^{-1}\left(c_{i} ; \phi\right)=\frac{\partial G\left(c_{i} ; \phi\right)}{\partial c_{i}}=\mu\left(c_{i}^{\beta_{1}}\left\{1-\left(1-c_{i}\right)^{\beta_{2}}\right\}^{\beta_{3}}\left\{\frac{\beta_{1}}{c_{i}}+\frac{\beta_{2} \beta_{3}\left(1-c_{i}\right)^{\beta_{2}-1}}{1-\left(1-c_{i}\right)^{\beta_{2}}}\right\}\right)
$$

From (ii), further differentiation of this function yields an expression for $f\left(z_{i} ; \phi\right)$. A value for $m_{i}^{(2)}\left(z_{i} ; \phi\right)$ can be obtained by numerically integrating the right side of (3.13).

If at the outset we begin by specifying a density function rather than a Lorenz curve, then whether estimation via (3.8) is tractable will depend on whether the cdf is invertible, either algebraically or computationally. For the GB2 distribution in (2.4), we can readily derive

$$
Z_{i}=F^{-1}\left(c_{i} ; \phi\right)=b\left(\frac{B^{-1}\left(c_{i} ; p, q\right)}{1-B^{-1}\left(c_{i} ; p, q\right)}\right)^{1 / a}
$$

and the GLC is given in (2.5). The remaining ingredient needed is the quantity $m_{i}^{(2)}$ which appears in the weight matrix. It is given by

$$
m_{i}^{(2)}=\frac{b^{2} \mathrm{~B}(p+2 / a, q-2 / a)}{\mathrm{B}(p, q)} B\left(B^{-1}\left(c_{i} ; p, q\right) ; p+2 / a, q-2 / a\right) \quad q>2 / a
$$

For a minimisation algorithm one can use any of several methods for implementing MD estimators, including a simple two-step, an iterative two-step, or a continuously updating estimator. We employ an iterative two-step estimator where in the first stage we find $\hat{\phi}_{L, 1}=\arg \min _{\phi} \mathbf{H}_{L}^{\prime}(\phi) \boldsymbol{\Omega}_{L, 1}^{-1} \mathbf{H}_{L}(\phi)$ with $\boldsymbol{\Omega}_{L, 1}=\mathbf{I}$ or some other pre-specified positive definite 
matrix. Using $\hat{\phi}_{L, 1}$ we compute $\Omega_{L, 2}=\Omega_{L}\left(\hat{\phi}_{L, 1}\right)$, then, in the second stage, we find $\hat{\boldsymbol{\phi}}_{L, 2}=\arg \min _{\phi} \mathbf{H}_{L}^{\prime}(\phi) \boldsymbol{\Omega}_{L, 2}^{-1} \mathbf{H}_{L}(\phi)$. An estimate for the covariance matrix for $\hat{\boldsymbol{\phi}}_{L}$ is given by

$$
\operatorname{var}\left(\hat{\phi}_{L}\right)=\frac{1}{T}\left(\frac{\partial \hat{\mathbf{H}}_{L}^{\prime}}{\partial \boldsymbol{\phi}} \hat{\mathbf{W}}_{L} \frac{\partial \hat{\mathbf{H}}_{L}}{\partial \phi^{\prime}}\right)^{-1}
$$

Computationally, it is convenient if we can obtain a closed form solution for the inverse $\mathbf{W}_{L}=\Omega_{L}^{-1}$, and simplify computations in other ways, particularly when we have a large number of groups. In Section 5 we derive a closed form expression for $\mathbf{W}_{L}$.

\subsection{Estimation with unknown $\tilde{\mathbf{z}}$}

If the data source does not include the group bounds, then we can estimate $\phi$ by considering only the second set of equations in (3.10), from which we obtain the estimator

$$
\hat{\boldsymbol{\phi}}_{L}^{*}=\arg \min _{\phi}\left(\tilde{\mathbf{y}}_{L}-\mathbf{G}(\mathbf{c} ; \phi)\right)^{\prime} \boldsymbol{\Omega}_{L, 22}^{-1}\left(\tilde{\mathbf{y}}_{L}-\mathbf{G}(\mathbf{c} ; \phi)\right)
$$

In the first iteration of the computations, a prespecified positive definite matrix is used in place of $\boldsymbol{\Omega}_{L, 22}^{-1}$. Then, with the aid of Lemma 1, in subsequent iterations estimates of $\phi$ from the previous iteration are used to find values for $z_{i}, m_{i}^{(2)}$ and $G\left(c_{i}\right)$, which in turn are used to update $\phi$. An estimate of the asymptotic covariance matrix for $\hat{\phi}_{L}^{*}$ is

$$
\operatorname{var}\left(\hat{\boldsymbol{\phi}}_{L}^{*}\right)=\frac{1}{T}\left(\frac{\partial \hat{\mathbf{G}}^{\prime}}{\partial \phi} \hat{\mathbf{\Omega}}_{L, 22}^{-1} \frac{\partial \hat{\mathbf{G}}}{\partial \phi^{\prime}}\right)^{-1}
$$

As expected, this estimator is asymptotically less efficient than that in equation (3.8) where more information is used.

\subsection{Comparison with conventional estimation methods}

The estimator in (3.16) provides a context useful for examining most conventional methods for estimating Lorenz curves. When least squares is used to regress income shares against a Lorenz function of population shares, the estimation problem can be written as 


$$
\min _{\phi} \sum_{i=1}^{N}\left\{\tilde{y}_{i}-G\left(c_{i} ; \phi\right)\right\}^{2}
$$

The consequences of using (3.18), ignoring that observations on cumulative proportions are correlated and heteroskedastic, are an inefficient estimator for $\phi$, and an incorrect covariance matrix for making inferences about $\phi^{13}$. The estimators from (3.18) and (3.16) are identical if $\Omega_{L, 22}^{-1}=\mathbf{I}$. Thus, minimising (3.18) yields a consistent estimator for $\phi$. However, the diagonal elements in $\Omega_{L, 22}^{-1}$ are substantially different, and, as is shown in our simulations and examples described in Sections 6 and 7, ignoring the inefficiency can make a difference.

The correct covariance matrix for the least-squares estimator for $\phi$ defined in (3.18) can be obtained from minimum distance estimation theory. We note that

$$
\hat{\phi}=\arg \min _{\phi}\left(\tilde{\mathbf{y}}_{L}-\mathbf{G}(\mathbf{c} ; \phi)\right)^{\prime} \boldsymbol{\Xi}\left(\tilde{\mathbf{y}}_{L}-\mathbf{G}(\mathbf{c} ; \phi)\right)
$$

is consistent and asymptotically normal with covariance matrix estimated by

$$
\operatorname{var}(\hat{\phi})=\frac{1}{T}\left\{\left(\frac{\partial \hat{\mathbf{G}}^{\prime}}{\partial \phi} \boldsymbol{\Xi} \frac{\partial \hat{\mathbf{G}}}{\partial \phi^{\prime}}\right)^{-1} \frac{\partial \hat{\mathbf{G}}^{\prime}}{\partial \phi} \boldsymbol{\Xi} \boldsymbol{\Omega}_{L, 22}(\hat{\phi}) \boldsymbol{\Xi} \frac{\partial \hat{\mathbf{G}}}{\partial \phi^{\prime}}\left(\frac{\partial \hat{\mathbf{G}}^{\prime}}{\partial \boldsymbol{\phi}} \boldsymbol{\Xi} \frac{\partial \hat{\mathbf{G}}}{\partial \phi^{\prime}}\right)^{-1}\right\}
$$

In the least-squares case where $\boldsymbol{\Xi}=\mathbf{I}$, this reduces to

$$
\operatorname{var}(\hat{\phi})=\frac{1}{T}\left\{\left(\frac{\partial \hat{\mathbf{G}}^{\prime}}{\partial \phi} \frac{\partial \hat{\mathbf{G}}}{\partial \phi^{\prime}}\right)^{-1} \frac{\partial \hat{\mathbf{G}}^{\prime}}{\partial \phi} \boldsymbol{\Omega}_{L, 22}(\hat{\phi}) \frac{\partial \hat{\mathbf{G}}}{\partial \phi^{\prime}}\left(\frac{\partial \hat{\mathbf{G}}^{\prime}}{\partial \phi} \frac{\partial \hat{\mathbf{G}}}{\partial \phi^{\prime}}\right)^{-1}\right\}
$$

It is also informative to put the "balanced fit” estimation procedure proposed by Wang et al (2011) within the context of the minimum distance estimator in (3.8) where information on both the Lorenz ordinates and the quantile function is used. They recommend minimising

$$
b \sum_{i=1}^{N-1}\left\{\tilde{L}_{i}-L\left(c_{i} ; \phi\right)\right\}^{2}+(1-b) \sum_{i=1}^{N-1}\left\{\tilde{z}_{i}-\mu \frac{\partial L\left(c_{i} ; \phi\right)}{\partial c_{i}}\right\}^{2}
$$

\footnotetext{
${ }^{13}$ Examples of using least squares are Kakwani (1980), Basmann et al. (1990), Chotikapanich (1993), Ryu and Slottje (1996), Datt (1998), and the World Bank website PovcalNet.
} 
where $0 \leq b \leq 1$ is a prespecified constant. Within our GLC framework in (3.8), this approach is equivalent to setting $\mathbf{W}_{L, 11}=b \mathbf{I}, \mathbf{W}_{L, 22}=(1-b) \mathbf{I}$ and $\mathbf{W}_{L, 12}=\mathbf{0}$. It is less efficient than using the optimal weight matrix. The relevant standard errors can be found by adapting the covariance matrix in (3.20) to the case where both sets of information are used. ${ }^{14,15}$

\section{Estimation for DGP2, stochastic $c_{i}$ and fixed $z_{i}$}

When the group boundaries $\mathbf{z}$ can be viewed as having been set prior to sampling, the random quantities are the cumulative proportions $\tilde{\mathbf{c}}$ and the average cumulative incomes $\tilde{\mathbf{y}}_{m}$. Results for this DGP, using single rather than cumulative proportions, have been provided by Hajargasht et al. (2012). Here, we extend these results to cumulative proportions. By doing so we are able to establish a relationship between the covariance matrices for the DGP1 and DGP2 estimators, and provide useful computational tools for the DGP1 estimator. Let $\mathbf{F}(\mathbf{z} ; \phi)$ and $\mathbf{m}(\mathbf{z} ; \phi)$ be $(N-1)$ and $N$-dimensional vectors, respectively, with $i$-th elements $F\left(z_{i} ; \phi\right)$ and $m\left(z_{i} ; \phi\right)$. Recognising that $\tilde{\mathbf{c}}$ is an estimator for $\mathbf{F}(\mathbf{z} ; \phi)$ and $\tilde{\mathbf{y}}_{m}$ is an estimator for $\mathbf{m}(\mathbf{z} ; \phi)$, we provide the moment conditions, the optimal weight matrix, and a GMM strategy for estimating either $\theta^{\prime}=\left(\mathbf{z}^{\prime}, \phi^{\prime}\right)$, or, if the group boundaries are provided, just $\phi$. Let $g_{i}(y)$ be an indicator function such that $g_{i}(y)=1$ if $0<y \leq z_{i}$ and $g_{i}(y)=0$ otherwise, then the cumulative proportions and the average cumulative incomes can be written respectively as

$$
\tilde{c}_{i}=\frac{1}{T} \sum_{t=1}^{T} g_{i}\left(y_{t}\right) \quad \tilde{y}_{m, i}=\frac{1}{T} \sum_{t=1}^{T} y_{t} g_{i}\left(y_{t}\right)
$$

The population moments corresponding to these sample moments are, respectively,

\footnotetext{
${ }^{14}$ Wang et al (2011) use bootstrapping to obtain standard errors.

${ }^{15}$ A further estimation method, not based on the nonlinear least squares estimator in (3.20) or modifications of it, was suggested by Castillo et al. (1998) and Sarabia et al. (1999). Suppose $\phi$ is of dimension $r$. They advocate using $r$ groups to solve for $\phi$ from the equations $\tilde{L}_{i}-L\left(c_{i} ; \phi\right)$, repeating the process for all $\left(\begin{array}{l}N \\ r\end{array}\right)$ combinations of groups, and selecting the median solutions for each of the elements in $\phi$ as the final estimator.
} 


$$
\begin{aligned}
& F\left(z_{i} ; \phi\right)=E\left[g_{i}(y)\right]=\int_{0}^{\infty} g_{i}(y) f(y) d y=\int_{0}^{z_{i}} f(y) d y \\
& m\left(z_{i} ; \phi\right)=E\left[y g_{i}(y)\right]=\int_{0}^{\infty} y g_{i}(y) f(y) d y=\int_{0}^{z_{i}} y f(y) d y .
\end{aligned}
$$

Suppose that the group limits are unknown and are treated as parameters to be estimated, with the complete set of unknown parameters given by $\theta_{0}^{\prime}=\left(\mathbf{z}^{\prime}, \phi_{0}^{\prime}\right)$. Under standard regularity conditions, the GMM estimator $\hat{\boldsymbol{\theta}}$ given by

$$
\hat{\boldsymbol{\theta}}=\arg \min _{\boldsymbol{\theta}} \mathbf{H}_{m}^{\prime}(\boldsymbol{\theta}) \tilde{\mathbf{W}}_{m} \mathbf{H}_{m}(\boldsymbol{\theta})
$$

(i) is a consistent estimator of $\theta_{0}$, and

(ii) $\quad \sqrt{T}\left(\hat{\boldsymbol{\theta}}-\boldsymbol{\theta}_{0}\right) \stackrel{d}{\longrightarrow} N\left(\mathbf{0}, \mathbf{V}_{m}\right) \quad$ where $\quad \mathbf{V}_{m}=\left(\frac{\partial \mathbf{H}_{m}^{\prime}}{\partial \boldsymbol{\theta}} \mathbf{W}_{m} \frac{\partial \mathbf{H}_{m}}{\partial \boldsymbol{\theta}^{\prime}}\right)^{-1}$

$$
\mathbf{H}_{m}(\boldsymbol{\theta})=\left[\begin{array}{c}
\tilde{\mathbf{c}}-\mathbf{F}(\boldsymbol{\theta}) \\
\tilde{\mathbf{y}}_{m}-\mathbf{m}(\boldsymbol{\theta})
\end{array}\right]
$$

$\mathbf{W}_{m}=\boldsymbol{\Omega}_{m}^{-1}\left(\boldsymbol{\theta}_{0}\right), \tilde{\mathbf{W}}_{m}=\boldsymbol{\Omega}_{m}^{-1}(\tilde{\boldsymbol{\theta}})$, where $\tilde{\boldsymbol{\theta}}$ is any consistent estimator for $\boldsymbol{\theta}_{0}$, and $\boldsymbol{\Omega}_{m}$ is the covariance matrix of the limiting distribution of $\sqrt{T} \mathbf{H}_{m}$. Closed form expressions for $\boldsymbol{\Omega}_{m}(\boldsymbol{\theta})$ and $\mathbf{W}_{m}$ are provided in Appendix B. If the group limits are observed, the same result holds, but with $\boldsymbol{\theta}$ replaced by $\phi$.

If the starting point is specification of a parametric income distribution, then the estimator in (4.4) will be tractable providing we have a satisfactory way for computing values of its cdf and its first and $2^{\text {nd }}$ moment distribution functions. That is, $F_{i}, m_{i}$ and $m_{i}^{(2)}$ can be readily calculated. Such is the case for the GB2 distribution, for example. Whether or not estimation is tractable when the starting point is a parametric Lorenz curve will depend on whether the cdf can be obtained by inverting the quantile function. The quantile function given 
in (3.14) for the Sarabia GLC cannot be readily inverted such that $c_{i}$ is written as a function of $Z_{i}$, making estimation intractable for this case.

\section{Some relationships between DGP1 and DGP2}

In Proposition 1 of this Section we give expressions for the relationship between the weight matrices for the two estimation procedures, expressions that can be utilised to compute the weight matrices for estimation under DGP1 from the weight matrix under DGP2. Let $\hat{\theta}$ from DGP2 be partitioned as $\hat{\theta}^{\prime}=\left(\hat{\mathbf{z}}^{\prime}, \hat{\phi}_{m}\right)$. In Proposition 2, we show that the asymptotic variances for the two estimators $\hat{\phi}_{L}$ and $\hat{\phi}_{m}$ are the same. Both propositions rely on the assumption that the a priori groupings from both methods are equivalent in the sense that $\mathbf{z}=\mathbf{F}^{-1}(\mathbf{c} ; \phi) .{ }^{16}$

In what follows, we use the following notation: if $\mathbf{a}^{\prime}=\left(a_{1}, \ldots, a_{n}\right)$ and $\mathbf{b}^{\prime}=\left(b_{1}, \ldots, b_{n}\right)$, then $(\mathbf{a . b})^{\prime}=\left(a_{1} b_{1}, \ldots, a_{n} b_{n}\right),(\mathbf{a} / \mathbf{b})^{\prime}=\left(a_{1} / b_{1}, \ldots, a_{n} / b_{n}\right)$, and $D[\mathbf{a}]$ is a diagonal matrix with the elements of $\mathbf{a}$ on the diagonal. Also, we use $\mathbf{j}^{\prime}=(1, \ldots, 1)$ to denote a vector of ones.

Proposition 1: Suppose group bounds $\mathbf{z}$ in DGP2 and population shares $\mathbf{c}$ in DGP1 are chosen in a way that $\mathbf{z}=\mathbf{F}^{-1}(\mathbf{c} ; \phi)$, then ${ }^{17}$

$$
\begin{aligned}
& \text { (i) } \boldsymbol{\Omega}_{L}=\mathbf{A} \boldsymbol{\Omega}_{m} \mathbf{A}^{\prime} \\
& \text { with } \quad \mathbf{A}=\left(\begin{array}{cc}
D[-\mathbf{j} / \mathbf{f}(\mathbf{z})]_{N-1} & \mathbf{0}_{(N-1) \times N} \\
{\left[\begin{array}{c}
-D[\mathbf{z}]_{N-1} \\
\mathbf{0}_{1 \times(N-1)}
\end{array}\right]} & \mathbf{I}_{N}
\end{array}\right) \\
& \text { (ii) } \left.\quad \mathbf{W}_{L}=\mathbf{A}^{\prime-1} \mathbf{W}_{m} \mathbf{A}^{-1} \quad \text { with } \quad \mathbf{A}^{-1}=\left(\begin{array}{cc}
D[-\mathbf{f}(\mathbf{z})]_{N-1} & \mathbf{0}_{(N-1) \times N} \\
D[-\mathbf{f}(\mathbf{z}) \cdot \mathbf{z}]_{N-1} \\
\mathbf{0}_{1 \times(N-1)}
\end{array}\right] \quad \mathbf{I}_{N}\right)
\end{aligned}
$$
and the lower diagonal blocks of $\mathbf{W}_{L}$ and $\mathbf{W}_{m}$ are equal.

\footnotetext{
${ }^{16}$ Note that, after sampling, the resulting grouped data will be different in the two cases even if the grouping is made on the same random sample.

17 The result in (i) might also be of interest to distribution free studies since the lower diagonal block provides the relationship between the variances of the GLC ordinates under the two DGPs, $\tilde{\mathbf{y}}_{L}$ and $\tilde{\mathbf{y}}_{m}$.
} 


$$
\begin{aligned}
& \boldsymbol{\Omega}_{L, 22}^{-1}=\left(\mathbf{B} \boldsymbol{\Omega}_{m} \mathbf{B}^{\prime}\right)^{-1}=\left(\mathbf{B B}^{\prime}\right)^{-1} \mathbf{B}\left[\mathbf{W}_{m}-\mathbf{W}_{m} \mathbf{C}\left(\mathbf{C}^{\prime} \mathbf{W}_{m} \mathbf{C}\right)^{-1} \mathbf{C}^{\prime} \mathbf{W}_{m}\right] \mathbf{B}^{\prime}\left(\mathbf{B B}^{\prime}\right)^{-1} \\
& \text { with } \mathbf{B}=\left(\left[\begin{array}{c}
-D[\mathbf{z}]_{N-1} \\
\mathbf{0}_{1 \times(N-1)}
\end{array}\right] \quad \mathbf{I}_{N}\right) \quad \text { and } \quad \mathbf{C}^{\prime}=\left[\begin{array}{lll}
\mathbf{I}_{N-1} & D[\mathbf{z}]_{N-1} & \mathbf{0}_{(N-1) \times 1}
\end{array}\right]
\end{aligned}
$$

Proof: See Appendix C.

Result (ii) provides a closed form for the weight matrix for DGP1 when both quantile and Lorenz equations are considered. Result (iii) provides a tractable formula for the weight matrix when only the Lorenz equations are considered. Its tractability comes from the facts that $\mathbf{B B}^{\prime}$ is diagonal, $\mathbf{C}^{\prime} \mathbf{W}_{m} \mathbf{C}$ is tri-diagonal and there are special formulae for inverting such matrices.

\section{Proposition 2}

(i) Known group bounds: If $z_{i}=F^{-1}\left(c_{i} ; \phi_{0}\right)$, then the asymptotic variance of $\hat{\phi}_{L}$ under DGP1 with observed $\left\{c_{i}, \tilde{y}_{L, i}, \tilde{z}_{i}\right\}$ is equal to asymptotic variance of $\hat{\phi}_{m}$ under DGP2 with observed $\left\{\tilde{c}_{i}, \tilde{y}_{m, i}, z_{i}\right\}$. That is,

$$
\frac{1}{T}\left(\left[\begin{array}{ll}
\frac{\partial \mathbf{F}^{\prime-1}}{\partial \boldsymbol{\phi}} & \frac{\partial \mathbf{G}^{\prime}}{\partial \boldsymbol{\phi}}
\end{array}\right] \mathbf{W}_{L}(\phi)\left[\begin{array}{c}
\partial \mathbf{F}^{-1} / \partial \boldsymbol{\phi}^{\prime} \\
\partial \mathbf{G} / \partial \boldsymbol{\phi}^{\prime}
\end{array}\right]\right)^{-1}=\frac{1}{T}\left(\left[\begin{array}{ll}
\frac{\partial \mathbf{F}^{\prime}}{\partial \phi} & \frac{\partial \mathbf{m}^{\prime}}{\partial \phi}
\end{array}\right] \mathbf{W}_{m}(\phi)\left[\begin{array}{c}
\partial \mathbf{F} / \partial \phi^{\prime} \\
\partial \mathbf{m} / \partial \boldsymbol{\phi}^{\prime}
\end{array}\right]\right)^{-1}
$$

where both are evaluated at $\phi_{0}$.

(ii) Unobserved group bounds: The asymptotic variance of $\hat{\phi}_{L}$ under DGP1 with observed $\left\{c_{i}, \tilde{y}_{i}\right\}$ is equal to the asymptotic variance of $\hat{\phi}_{m}$ under DGP2 with observed $\left\{\tilde{c}_{i}, \tilde{y}_{i}\right\}$. That is, $\frac{1}{T}\left(\frac{\partial \mathbf{G}^{\prime}}{\partial \phi} \mathbf{\Omega}_{L, 22}^{-1}(\phi) \frac{\partial \mathbf{G}}{\partial \phi^{\prime}}\right)^{-1}$ evaluated at $\phi_{0}$ is equal to the lower diagonal block of $\operatorname{cov}(\hat{\boldsymbol{\theta}})=\frac{1}{T}\left(\left[\begin{array}{ll}\frac{\partial \mathbf{F}^{\prime}}{\partial \boldsymbol{\theta}} & \frac{\partial \mathbf{m}^{\prime}}{\partial \boldsymbol{\theta}}\end{array}\right] \mathbf{W}_{m}(\boldsymbol{\theta})\left[\begin{array}{l}\partial \mathbf{F} / \partial \boldsymbol{\theta}^{\prime} \\ \partial \mathbf{m} / \partial \boldsymbol{\theta}^{\prime}\end{array}\right]\right)^{-1}$ evaluated at $\boldsymbol{\theta}_{0}^{\prime}=\left(\mathbf{F}^{-1}\left(\mathbf{c}, \phi_{0}\right)^{\prime}, \phi_{0}^{\prime}\right)$

Proof: See Appendix C. 
The following lemma is used to prove the above proposition. We include it here because of its potential for more general use.

Lemma 2: If $c_{i}=F\left(z_{i} ; \phi\right)$, then under suitable differentiability conditions,

(i) $\frac{\partial F\left(z_{i} ; \phi\right)}{\partial \phi_{j}}=-\frac{\partial F^{-1}\left(c_{i} ; \phi\right)}{\partial \phi_{j}} / \frac{\partial F^{-1}\left(c_{i} ; \phi\right)}{\partial c_{i}}=-f\left(z_{i} ; \phi\right) \frac{\partial F^{-1}\left(c_{i} ; \phi\right)}{\partial \phi_{j}}=-\frac{\partial^{2} G\left(c_{i} ; \phi\right)}{\partial c_{i} \partial \phi_{j}} / \frac{\partial^{2} G\left(c_{i} ; \phi\right)}{\partial c_{i}^{2}}$

(ii) $\frac{\partial m\left(z_{i} ; \phi\right)}{\partial \phi_{j}}=\frac{\partial G\left(c_{i} ; \phi\right)}{\partial \phi_{j}}-\frac{\partial G\left(c_{i} ; \phi\right)}{\partial c_{i}} \frac{\partial^{2} G\left(c_{i} ; \phi\right) / \partial c_{i} \partial \phi_{j}}{\partial^{2} G\left(c_{i} ; \phi\right) / \partial c_{i}^{2}}$

(iii) $\quad \frac{\partial G\left(c_{i} ; \phi\right)}{\partial \phi_{j}}=\frac{\partial m\left(z_{i} ; \phi\right)}{\partial \phi_{j}}-z_{i} \frac{\partial F\left(z_{i} ; \phi\right)}{\partial \phi_{j}}$

Proof: (i) can be obtained by differentiating $F\left(F^{-1}\left(c_{i} ; \phi\right) ; \phi\right)=c_{i}$ with respect to $\phi_{j}$, applying the chain rule and the results from Lemma 1; (ii) is obtained by differentiating $m\left(z_{i} ; \phi\right)=G\left(F\left(z_{i}, \phi\right) ; \phi\right)$ with respect to $\phi_{j}$, applying the chain rule and the result from (i); and (iii) is obtained by differentiating $G\left(c_{i} ; \phi\right)=m\left(F^{-1}\left(c_{i} ; \phi\right) ; \phi\right)$ with respect to $\phi_{j}$, applying the chain rule and the result from (i).

\section{Monte Carlo experiments}

Monte Carlo experiments were carried out to address the following questions: (i) Do the estimators perform well? (ii) Does the method of grouping matter? (iii) How do the various estimators compare with each other and with some traditional Lorenz curve estimation methods? (iv) How do the estimators perform in finite samples? Details of the experiments and the results are given in Appendix E. In all cases there were 500 replications of samples of size 10,000. The following conclusions were drawn.

1. The covariance matrices $\Omega_{L, 22}$ and $\Omega_{m, 22}$, computed at true values, computed at sample values, and estimated from the simulated draws, were all very similar, indicating that they are an accurate reflection of the variation in the GLC ordinates. 
2. The inverses of the covariance matrices (the weight matrices for both DGPs), are starkly different from the identity matrix often used implicitly in least squares estimation.

3. Using DGP1 the parameters of a GB2 distribution were accurately estimated for both known and unknown group boundaries. As expected, knowing the group boundaries provides more efficient estimates, but the differences are not large. Also, there are only minor differences between (i) the asymptotic variances computed at the true parameter values, (ii) the average of the estimated variances over the replications, and (iii) the Monte Carlo sample variance of the estimated parameters.

4. The conclusions for DGP2 are similar to those for DGP1. One difference is that when the group boundaries are unknown, they are estimated. However, they are estimated very accurately and there is only a small loss in efficiency of estimation of the GB2 parameters. The asymptotic equivalence of estimation under DGP1 and DGP2, described in Proposition 2, is confirmed.

5. A comparison of minimum distance estimation with naive least squares, for both GB2 parameters and SCS-GLC parameters, revealed substantial gains in efficiency from minimum distance estimation. Moreover, least-squares variance estimates were poor, mainly because the least squares estimates sometimes approached values that yield an infinite second moment.

\section{World Bank estimation of Lorenz curves}

The World Bank collects and publishes annual grouped income or expenditure data for many countries and years. Historically, the number of population and income share groups has been between 10 and $20 .{ }^{18}$ These data are used to estimate Lorenz curves and, based on the estimates,

\footnotetext{
${ }^{18}$ Recently, the World Bank has started to publish data in the form of 100 groups and to base inequality and poverty estimates on these groups. Earlier groups and their Lorenz curve estimations are still present on the PovcalNet website, however.
} 
a variety of poverty and inequality measures are provided. The Lorenz specifications that are used are the general quadratic (GQ) proposed by Villasenor and Arnold (1989) and the beta proposed by Kakwani (1980). ${ }^{19}$ On PovcalNet both of these curves are estimated and the one with the better fit is chosen to compute poverty and inequality measures. ${ }^{20}$ We first review the GQ and beta Lorenz curves and then estimate these models for several countries, comparing the results from the method employed by PovcalNet with those from our optimal estimation methods. The results are also compared with those from the GB2 and SCS specifications.

The GQ Lorenz curve depends on three parameters $\beta_{1}, \beta_{2}, \beta_{3}$ and can be written as

$$
L_{i}\left(1-L_{i}\right)=\beta_{1}\left(c_{i}^{2}-L_{i}\right)+\beta_{2} L_{i}\left(c_{i}-1\right)+\beta_{3}\left(c_{i}-L_{i}\right)
$$

The parameters are often estimated by replacing $L_{i}$ with the observed cumulative income proportions and applying least squares to equation (7.1). This practice is not innocuous since the $L_{i}$ 's are present on both sides of the equation. In the GLC framework that we have been using, (7.1) can be written as

$$
\tilde{y}_{i}=G\left(c_{i}\right)=-\frac{\mu}{2}\left(\beta_{2} c_{i}+e+\sqrt{m c_{i}^{2}+n c_{i}+e^{2}}\right)
$$

where $e=-\left(1+\beta_{1}+\beta_{2}+\beta_{3}\right), m=\beta_{2}^{2}-4 \beta_{1}$, and $n=2 \beta_{2} e-4 \beta_{3}$.

One problem with beginning a study with specification of a parametric Lorenz curve rather than a parametric income distribution is that the income distribution implied by the Lorenz curve may only be valid for a limited range of incomes. Having a finite support for income can be a serious drawback with potentially important implications since, in poverty and inequality analysis, the tails of the distribution play important roles. Not all Lorenz curves

\footnotetext{
${ }^{19}$ The literature abounds with proposals for parametric Lorenz curves. Examples are Kakwani and Podder (1976), Rasche et al. (1980), Basmann et al. (1990), Chotikapanich (1993), Ryu and Slottje (1996), Sarabia et al. (1999) and Wang et al (2011). The literature is surveyed by Sarabia (2008).

${ }^{20}$ See Datt(1998) for details of the World Bank approach.
} 
suffer from this problem; for example, the SCS curve does not, but it is an issue with the GQ. It can be shown that its support is given by

$$
y_{\min }=-\frac{\mu}{2}\left[\frac{n}{2 \sqrt{e^{2}}}+\beta_{2}\right] \quad \text { and } \quad y_{\max }=\frac{\mu}{2}\left[-\frac{(2 m+n)}{2 \sqrt{m+n+e^{2}}}-\beta_{2}\right]
$$

The second model used by the PovcalNet is the beta Lorenz curve

$$
L_{i}=c_{i}-\beta_{1} c_{i}^{\beta_{2}}\left(1-c_{i}\right)^{\beta_{3}} \text {, with } \quad 0<\beta_{1}, \beta_{2}, \beta_{3}<1
$$

Providing $0<\beta_{1}, \beta_{2}, \beta_{3}<1$, the corresponding distribution has an infinite support, but the second order moment becomes infinite if $\beta_{2}<0.5$ or $\beta_{3}<0.5$. In our examples, we frequently obtain estimates for $\beta_{3}$ that are less than 0.5 , even for countries with Gini coefficients as low as 0.3 .

In Table 1, we compare estimates obtained using the World Bank’s least squares methods with estimates obtained from our proposed minimum distance methods, for 5 selected countries and the following estimators.

(i) OLS-GQ: Least squares estimation applied to (7.1) - the World Bank approach.

(ii) MD-GQ: Minimum distance estimation applied to (7.2).

(iii) NLS-Beta: Nonlinear least squares applied to the GLC version of (7.3).

(iv) MD-Beta: Minimum distance estimation applied to the GLC in (iii).

(v) MD-GB2: Minimum distance estimation applied to the GLC in (2.5).

(vi) MD-SCS: Minimum distance estimation applied to the GLC in (2.3).

In addition to the parameter estimates and their standard errors, estimates for the Gini coefficient and the headcount ratio $H$ (using a poverty line of $\$ 38 /$ month) are reported. Because sample sizes are not provided on the PovcalNet website, we use $T=10,000$ to compute standard errors. Useful diagnostic information that is provided is the supports of the distributions $\left[y_{\min }, y_{\max }\right]$, the $J$-statistics for testing the validity of the estimating equations, and the percentage errors in predictions of the incomes of the first and last groups, $S_{1}$ and $S_{20}$. The 
data are from 2004 or 2005, extracted from the PovcalNet website where we obtained population and income shares for 20 groups and mean income for each country. In all cases the $z_{i}$ 's are not observed, DGP1 is assumed, and therefore only the second set of equations in (3.10) is considered. The results can be summarised as follows:

1. Estimates for $\beta_{1}, \beta_{2}, \beta_{3}$ from least squares and from MD estimation are often sufficiently different to have important implications, especially for the GQ model. The least-squares estimates for the GQ and beta Lorenz curves lead to relatively large percentage errors for the estimated incomes in the first group, $S_{1}$. This can lead to poor estimates for the headcount ratio and most likely even poorer estimates for other poverty measures. ${ }^{21}$ ChinaUrban and Iran are good examples. ${ }^{22}$ In these cases the minimum distance method improves the estimated support and provides more reasonable values for the head-count ratio, values which are not substantially different from those from GB2 or SCS.

2. The least-squares standard errors do not use (3.21), and so, although they are very different from the others, a direct comparison is not valid.

3. The GQ and especially the beta GLC can suffer from heavy-tail "inflexibility". For example, for Kenya, with a Gini coefficient of 0.47, the GQ second moment becomes infinite and so no MD-GQ estimates can be reported. With the beta GLC we have infinite second order moments for all cases except China Urban. The reported results are from estimating the model using $c_{N}=0.99999$ instead of 1 to ensure the existence of a finite second moment. ${ }^{23}$ There were no problems with the GB2 and SCS curves for the examples considered here, but for countries with higher degrees of inequality such as South Africa or Brazil, even these functions suffer from the same issue.

\footnotetext{
21 This has been recognized in PovcalNet and estimates from a beta Lorenz curve are used in such cases.

22 This problem also existed for a number of other countries. We have limited our reported results to 5 countries.

${ }^{23}$ Any finite positive definite weight matrix (instead of the optimal weight matrix $\mathbf{W}$ ) will give a consistent estimator for the parameters, but inference methods still need to be worked out for when the variance is infinite. Exposing this problem reinforces the need for a sound statistical framework to reveal the difficulty and to provide a foundation for investigating a solution.
} 
4. Unlike the poverty measures, estimates of the Gini coefficient are not overly sensitive to the method of estimation or the model used.

5. An overall measure of the goodness of fit of the models is the $J$-statistic given by $J=T\left(\tilde{\mathbf{y}}_{L}-\mathbf{G}(\mathbf{c} ; \hat{\phi})\right)^{\prime} \hat{\mathbf{\Omega}}_{L, 22}^{-1}\left(\tilde{\mathbf{y}}_{L}-\mathbf{G}(\mathbf{c} ; \hat{\phi})\right)$. Smaller values of $J$ indicate better fitting Lorenz curves. Using this criterion, GB2 performs better than SCS in three of the cases and is worse for the other two. They both have substantially smaller $J$-statistics compared to the GQ model ${ }^{24,25}$.

Based on our theoretical analyses, the Monte Carlo experiments, and the applications, we make the following recommendations to improve the current practice of Lorenz curve estimation by the World Bank and others.

1. Both the GQ and beta Lorenz curves have undesirable properties. The GQ has an inflexible bounded support resulting in poor estimates for poverty measures and the beta Lorenz curve has an "inflexible” upper tail. Other Lorenz curve candidates without such problems, such as the GB2 or SCS models, are preferable. These two models, although not linear, are still easy to estimate and exhibit good performance.

2. The proposed MD estimation method should be used instead of least squares methods since it is more efficient and provides sound standard errors. With the GQ model, the MD estimator provides better estimates for the support of the distribution and more accurate poverty measure estimates.

\footnotetext{
${ }^{24}$ Povcal decides between GQ and beta Lorenz curves based on their in-sample income share prediction performance where GQ often performs better. The $J$-stat criterion seems to support the beta Lorenz curve over GQ more often.

${ }^{25}$ If the moment conditions are valid, $J \sim \chi_{(N-d)}^{2}$ where $d$ is the number of estimated parameters and $N$ is the number of moment conditions. Because the assumed sample size is large $(T=10000)$, there is a strong chance of rejecting a null hypothesis that the moment conditions are valid, and so we prefer to use the $J$-statistic as an indication of goodness of fit rather than as a formal test statistic. For testing purposes, the $1 \%$ critical value for 16 degrees of freedom is 32 .
} 
3. It might be worthwhile to search for a Lorenz curve specification that can be made linear in parameters but still has good tail properties.

4. If possible, published data should include the sample size, and the variance and boundaries of the groups. Having this information makes parametric inference easier and inference using distribution-free approaches feasible.

\section{Conclusion}

The Lorenz curve has become one of the main tools for poverty and inequality analysis because of its theoretical appeal and because of data availability. However, so far methods for parametric estimation of Lorenz curves have been ad hoc. In this paper, we developed statistically sound approaches for using grouped data to estimate and make inferences about parametric Lorenz curves. We performed simulation experiments and real data examples to show that the proposed methods work well and can improve on conventional approaches. In particular we showed that World Bank’s estimates for poverty and inequality measures can be improved by using the proposed methods. There is also scope for extending the current work by incorporating features such as censoring, trimming, heavy tails, measurement errors, and survey sampling designs that are recognised to have important implications for welfare measurement [see e.g. Cowell and Victoria-Feser (2003, 2006, 2007, 2008), Cowell and Flachaire (2015) and references cited there in].

\section{Appendices}

The appendices are available as web-based supplementary material.

\section{Acknowledgements}

This research was supported by Australian Research Council Grant DP140100673. The authors are grateful to Jenny Williams whose comments on an earlier draft improved the exposition.

\section{References}

Anand, S., Segal, P. (2008). What do we know about global income inequality? Journal of Economic Literature 46:57-94. 
Basmann, R., Hayes, K., Slottje, D., Johnson J. (1990). A general functional form for approximating the Lorenz curve. Journal of Econometrics 43:77-90.

Beach, C., Davidson, R. (1983). Distribution-free statistical inference with Lorenz curves and income shares. Review of Economic Studies 50:723-725.

Bishop, J., Chakraborti, S., Thistle, P. (1989). Asymptotically distribution-free statistical inference for generalized Lorenz curves. Review of Economics and Statistics 71:725727.

Bourguignon, F., Morrisson, C. (2002). Inequality among world citizens: 1820-1992. American Economic Review 92:727-744.

Castillo, E., Hadi, A.S., Sarabia, J.M. (1998). A method for estimating Lorenz curves. Communications in Statistics, Theory and Methods 27:2037-2063.

Chen, S., Ravallion, M. (2011). The developing world is poorer than we thought, but no less successful in the fight against poverty. Quarterly Journal of Economics 125:1577-1625.

Chotikapanich, D. (1993). A comparison of alternative functional forms for the Lorenz curve. Economics Letters 41:129-138.

Chotikapanich, D., ed. (2008). Modeling Income Distributions and Lorenz Curves. New York: Springer.

Chotikapanich, D., Griffiths, W. (2002). Estimating Lorenz curves using a Dirichlet distribution. Journal of Business and Economic Statistics 20:290-295.

Chotikapanich, D., Griffiths, W., Prasada Rao, D., Valencia, V. (2012). Global income distributions and inequality, 1993 and 2000: Incorporating country level inequality modeled with beta distributions. Review of Economics and Statistics 94:52-73.

Cowell, F., Flachaire, E. (2015) Statistical methods for distributional analysis. In Atkinson, A.B., Bourguignon, F., eds., Handbook of Income Distribution, Vol 2A. Amsterdam: North Holland, pp.359-466.

Cowell, F., Victoria-Feser, M. (2002). Welfare rankings in the presence of contaminated data, Econometrica. 70:1221-1233.

Cowell F., Victoria-Feser, M. (2003). Distribution-free inference for welfare indices under complete and incomplete information. Journal of Economic Inequality 1(3):1-29.

Cowell F., Victoria-Feser, M. (2006). Distributional dominance with trimmed data. Journal of Business and Economics Statistics 24:291-300.

Cowell, F., Victoria-Feser, M. (2007). Robust stochastic dominance: A semi-parametric approach. Journal of Economic Inequality 5:21-37. 
Cowell, F., Victoria-Feser, M. (2008). Modelling Lorenz curves: Robust and semi-parametric issues. In Chotikapanich, D., ed., Modelling Income Distributions and Lorenz Curves. New York: Springer, pp.241-225.

Cressie N., Read, T. (1984). Multinomial goodness-of-fit tests. Journal of the Royal Statistical Society, Series B 46:440-464.

Datt, G. (1998). Computational tools for poverty measurement and analysis. Washington D.C.: International Food Policy Research Institute (IFPRI) Discussion Paper No.50 (Food Consumption and Nutrition Division).

Ferguson, T. S. (1958). A method of generating best asymptotically normal estimates with application to the estimation of bacterial densities. The Annals of Mathematical Statistics 29(4):1046-1062.

Gastwirth, J. (1971). A general definition of the Lorenz curve. Econometrica 39:1037-1039.

Gastwirth, J. (1972). The estimation of the Lorenz curve and Gini index. The Review of Economics and Statistics 54:306-316.

Gastwirth, J., Gail, M. (1985). Simple asymptotically distribution-free methods comparing Lorenz curves and Gini indices obtained from complete data. In Basmann R., Rhodes, G. Jr., eds., Advances in Econometrics 4. Stamford CT: JAI Press, pp. 229-243.

Goldie, C. (1977). Convergence theorems for empirical Lorenz curves and their inverses. Advances in Applied Probability 9:765-791.

Hajargasht, G., Griffiths, W., Brice, J., Rao, D. S. P., Chotikapanich, D. (2012). Inference for income distributions using grouped data. Journal of Business of Economic Statistics 30:563-575.

Hansen, L. P. (1982). Large sample properties of generalized method of moments estimators. Econometrica 50(4):1029-1054.

Jorda, V., Niño -Zarazúa, M. (2016). Global inequality: How large is the effect of top incomes? Technical report, WIDER Working Paper.

Kakwani, N., Podder, N. (1976). Efficient estimation of the Lorenz curve and associated inequality measures from grouped observations. Econometrica 44:137-148.

Kakwani, N. (1980). On a class of poverty measures. Econometrica 48:437-446.

Kleiber, C., Kotz, S. (2003). Statistical Size Distributions in Economics and Actuarial Sciences. New York: John Wiley and Sons.

Maasoumi, E. (1999). Empirical analyses of inequality and welfare. In Pesaran, M.H., Schmidt, P., eds., Handbook of Applied Econometrics, Vol. II: Microeconomics. Malden MA: Blackwell, pp.202-245. 
Newey W., McFadden, D. (1994). Large sample estimation and hypothesis testing. In Engle, R.F., McFadden, D.L., eds., Handbook of Econometrics, Vol.4. Amsterdam: Elsevier, pp.2113-2245.

Pinkovskiy, M., Sala-i-Martin, X. (2014). Africa is on time. Journal of Economic Growth 19:311-338.

Rasche, R., Gaffney, J., Koo, A., Obst, N. (1980). Functional forms for estimating the Lorenz curve. Econometrica 48:1061-1064.

Ryu, H., Slottje, D. (1996). Two flexible functional form approaches for approximating the Lorenz curve. Journal of Econometrics 72:251-274.

Sala-i Martin, X. (2006). The world distribution of income: falling poverty and convergence, period. The Quarterly Journal of Economics 121:351-397.

Sarabia, J. (2008). Parametric Lorenz curves: models and applications. In Chotikapanich, D., ed., Modelling Income Distributions and Lorenz Curves. New York: Springer, pp.167190.

Sarabia, J., Castillo, E., Slottje, D. (1999). An ordered family of Lorenz curves. Journal of Econometrics 91:43-60.

Villasenor, J., Arnold, B. (1989). Elliptical Lorenz curves. Journal of Econometrics 40:327338.

Victoria-Feser M. (2000). A general robust approach to the analysis of income distribution, inequality and poverty. International Statistical Review 68:277-293.

Victoria-Feser M., Ronchetti, E. (1997). Robust estimation for grouped data. Journal of the American Statistical Association 92:333-340.

Wang, Z., Ng, Y.-K., Smyth, R. (2011). A general method for creating Lorenz curves. The Review of Income and Wealth 57:561-582.

Wang, Z., Smyth, R. (2015). A hybrid method for creating Lorenz curves. Economics Letters 133:59-63.

Wu, X. (2006). Inference and density estimation with interval statistics. www.agecon2.tamu.edu/people/faculty/wu-ximing/agecon2/.../interval.pdf 
Table 1: A comparison of Lorenz curve estimation methods for selected countries

\section{China Urban}

\begin{tabular}{|c|c|c|c|c|c|c|c|c|c|c|c|}
\hline & \multicolumn{2}{|c|}{ OLS-GQ } & \multicolumn{2}{|c|}{ MD-GQ } & \multirow{2}{*}{$\begin{array}{c}\text { NLS-Beta } \\
\text { Par }\end{array}$} & \multicolumn{2}{|c|}{ MD-Beta } & \multicolumn{2}{|c|}{ MD-GB2 } & \multicolumn{2}{|c|}{ MD-SCS } \\
\hline & Par & SE & Par & SE & & Par & SE & Par & SE & Par & SE \\
\hline$\beta_{1} / p$ & 0.889 & 0.003 & 0.912 & 0.009 & 0.638 & 0.641 & 0.005 & 2.369 & 0.391 & -0.305 & 0.186 \\
\hline$\beta_{2} / q$ & -1.260 & 0.010 & -1.344 & 0.018 & 0.935 & 0.935 & 0.003 & 1.778 & 0.252 & 0.706 & 0.026 \\
\hline$\beta_{3} / a$ & 0.187 & 0.006 & 0.140 & 0.005 & 0.522 & 0.519 & 0.011 & 1.842 & 0.168 & 1.607 & 0.183 \\
\hline$\mu$ & NA & NA & 160.95 & 1.22 & 161.87 & 162.23 & 1.39 & 161.15 & 1.29 & 161.53 & 1.31 \\
\hline Gini & 0.347 & 0.000 & 0.349 & 0.004 & 0.348 & 0.351 & 0.004 & 0.345 & 0.004 & 0.347 & 0.004 \\
\hline$H$ & 0.004 & NA & 0.021 & 0.001 & 0.017 & 0.017 & 0.001 & 0.019 & 0.001 & 0.021 & 0.001 \\
\hline$y_{\min }$ & \multicolumn{2}{|c|}{37.06} & \multicolumn{2}{|c|}{31.80} & 0 & \multicolumn{2}{|c|}{0} & \multicolumn{2}{|c|}{0} & \multicolumn{2}{|c|}{0} \\
\hline$y_{\max }$ & \multicolumn{2}{|c|}{1495.86} & \multicolumn{2}{|c|}{1935.52} & $\infty$ & \multicolumn{2}{|c|}{$\infty$} & \multicolumn{2}{|c|}{$\infty$} & \multicolumn{2}{|c|}{$\infty$} \\
\hline$J$-Stat & \multicolumn{2}{|c|}{317.10} & \multicolumn{2}{|c|}{85.12} & 29.76 & \multicolumn{2}{|c|}{28.88} & \multicolumn{2}{|c|}{31.08} & \multicolumn{2}{|c|}{53.83} \\
\hline$S_{1}$ & \multicolumn{2}{|c|}{7.279} & \multicolumn{2}{|c|}{-2.169} & 0.631 & \multicolumn{2}{|c|}{-0.185} & \multicolumn{2}{|c|}{-0.505} & \multicolumn{2}{|c|}{-3.053} \\
\hline$S_{20}$ & \multicolumn{2}{|c|}{-0.873} & \multicolumn{2}{|c|}{0.022} & 0.820 & \multicolumn{2}{|c|}{2.057} & \multicolumn{2}{|c|}{-3.854} & \multicolumn{2}{|c|}{-2.030} \\
\hline
\end{tabular}

\section{Nigeria}

\begin{tabular}{|c|c|c|c|c|c|c|c|c|c|c|c|}
\hline & \multicolumn{2}{|c|}{ OLS-GQ } & \multicolumn{2}{|c|}{ MD-GQ } & \multirow{2}{*}{$\begin{array}{c}\text { NLS-Beta } \\
\text { Par }\end{array}$} & \multicolumn{2}{|c|}{ MD-Beta } & \multicolumn{2}{|c|}{ MD-GB2 } & \multicolumn{2}{|c|}{ MD-SCS } \\
\hline & Par & SE & Par & SE & & Par & SE & Par & SE & Par & SE \\
\hline$\beta_{1} / p$ & 0.979 & 0.003 & 0.999 & 0.010 & 0.780 & 0.762 & 0.005 & 4.880 & 1.508 & -1.983 & 0.521 \\
\hline$\beta_{2} / q$ & -0.932 & 0.015 & -1.068 & 0.026 & 0.978 & 0.960 & 0.002 & 5.202 & 1.617 & 0.811 & 0.024 \\
\hline$\beta_{3} / a$ & 0.189 & 0.006 & 0.130 & 0.005 & 0.549 & 0.511 & 0.011 & 0.893 & 0.150 & 3.367 & 0.516 \\
\hline$\mu$ & NA & NA & 39.76 & 0.33 & 40.05 & 40.84 & 0.43 & 39.98 & 0.36 & 40.01 & 0.35 \\
\hline Gini & 0.400 & 0.000 & 0.401 & 0.003 & 0.401 & 0.413 & 0.005 & 0.400 & 0.003 & 0.401 & 0.003 \\
\hline$H$ & 0.619 & NA & 0.618 & 0.004 & 0.613 & 0.614 & 0.004 & 0.621 & 0.004 & 0.626 & 0.004 \\
\hline$y_{\min }$ & \multicolumn{2}{|c|}{6.13} & \multicolumn{2}{|c|}{4.88} & 0 & \multicolumn{2}{|c|}{0} & \multicolumn{2}{|c|}{0} & \multicolumn{2}{|c|}{0} \\
\hline$y_{\max }$ & \multicolumn{2}{|c|}{289.22} & \multicolumn{2}{|c|}{326.87} & $\infty$ & \multicolumn{2}{|c|}{$\infty$} & \multicolumn{2}{|c|}{$\infty$} & \multicolumn{2}{|c|}{$\infty$} \\
\hline$J$-Stat & \multicolumn{2}{|c|}{429.16} & \multicolumn{2}{|c|}{95.16} & 240.03 & \multicolumn{2}{|c|}{57.46} & \multicolumn{2}{|c|}{38.70} & \multicolumn{2}{|c|}{66.80} \\
\hline$S_{1}$ & \multicolumn{2}{|c|}{12.680} & \multicolumn{2}{|c|}{-2.473} & 14.408 & \multicolumn{2}{|c|}{0.224} & \multicolumn{2}{|c|}{-0.113} & \multicolumn{2}{|c|}{-4.124} \\
\hline$S_{20}$ & \multicolumn{2}{|c|}{-0.520} & \multicolumn{2}{|c|}{-0.188} & 2.252 & \multicolumn{2}{|c|}{11.554} & \multicolumn{2}{|c|}{-1.024} & \multicolumn{2}{|c|}{-0.541} \\
\hline
\end{tabular}

Pakistan

\begin{tabular}{cccccccccccc}
\hline & \multicolumn{2}{c}{ OLS-GQ } & \multicolumn{2}{c}{ MD-GQ } & NLS-Beta & \multicolumn{2}{c}{ MD-Beta } & \multicolumn{2}{c}{ MD-GB2 } & \multicolumn{2}{c}{ MD-SCS } \\
\hline & Par & SE & Par & SE & Par & Par & SE & Par & SE & Par & SE \\
\hline$\beta_{1} / p$ & 0.752 & 0.010 & 0.851 & 0.010 & 0.552 & 0.537 & 0.005 & 1.565 & 0.206 & 0.005 & 0.117 \\
$\beta_{2} / q$ & -1.267 & 0.029 & -1.455 & 0.016 & 0.946 & 0.918 & 0.003 & 0.691 & 0.067 & 0.640 & 0.027 \\
$\beta_{3} / a$ & 0.269 & 0.018 & 0.167 & 0.005 & 0.479 & 0.403 & 0.014 & 3.720 & 0.276 & 1.183 & 0.115 \\
$\mu$ & NA & NA & 63.800 & 0.492 & 65.800 & 68.272 & 0.787 & 66.096 & 0.566 & 65.744 & 0.543 \\
Gini & 0.312 & 0.001 & 0.305 & 0.004 & 0.312 & 0.337 & 0.007 & 0.315 & 0.035 & 0.311 & 0.004 \\
$H$ & 0.232 & NA & 0.236 & 0.004 & 0.231 & 0.219 & 0.004 & 0.216 & 0.004 & 0.216 & 0.004 \\
$y_{\min }$ & 23.48 & 18.94 & 0 & & 0 & & 0 & & 0 & \\
$y_{\max }$ & 1618.31 & 1432.34 & & & & & & & & & \\
$J$-Stat & 2893.60 & 368.90 & 215.86 & 36.74 & & 20.22 & & 15.59 \\
$S_{1}$ & 13.322 & -3.091 & 8.414 & -0.136 & & 0.222 & & 0.207 \\
$S_{20}$ & 2.362 & -6.105 & 2.085 & 22.952 & & 3.770 & & -0.020 \\
\hline
\end{tabular}




\begin{tabular}{|c|c|c|c|c|c|c|c|c|c|c|c|}
\hline \multicolumn{12}{|c|}{ Kenya } \\
\hline & \multicolumn{2}{|c|}{ OLS-GQ } & \multicolumn{2}{|c|}{ MD-GQ } & \multirow{2}{*}{$\frac{\text { NLS-Beta }}{\text { Par }}$} & \multicolumn{2}{|c|}{ MD-Beta } & \multicolumn{2}{|c|}{ MD-GB2 } & \multicolumn{2}{|c|}{ MD-SCS } \\
\hline & Par & SE & Par & SE & & Par & SE & Par & SE & Par & SE \\
\hline$\beta_{1} / p$ & 0.755 & 0.008 & \multirow{4}{*}{\multicolumn{2}{|c|}{$\begin{array}{l}\text { Infinite second } \\
\text { order moment } \\
\text { No estimates } \\
\text { are reported }\end{array}$}} & 0.808 & 0.779 & 0.005 & 1.337 & 0.180 & -0.049 & 0.147 \\
\hline$\beta_{2} / q$ & -0.485 & 0.048 & & & 0.983 & 0.953 & 0.002 & 1.015 & 0.133 & 0.546 & 0.033 \\
\hline$\beta_{3} / a$ & 0.222 & 0.016 & & & 0.417 & 0.326 & 0.014 & 1.970 & 0.172 & 1.443 & 0.142 \\
\hline$\mu$ & NA & NA & & & 65.506 & 71.580 & 1.698 & 66.184 & 1.355 & 65.521 & 1.090 \\
\hline Gini & 0.456 & 0.010 & & & 0.477 & 0.520 & 0.010 & 0.482 & 0.009 & 0.477 & 0.007 \\
\hline$H$ & 0.438 & NA & & & 0.441 & 0.438 & 0.004 & 0.437 & 0.004 & 0.439 & 0.005 \\
\hline$y_{\min }$ & \multicolumn{2}{|c|}{9.75} & & & 0 & \multicolumn{2}{|c|}{0} & \multicolumn{2}{|c|}{0} & \multicolumn{2}{|c|}{0} \\
\hline$y_{\max }$ & \multicolumn{2}{|c|}{3546.00} & & & & & & \multicolumn{2}{|c|}{$\infty$} & \multicolumn{2}{|c|}{$\infty$} \\
\hline$J$-Stat & \multicolumn{2}{|c|}{ NA } & & & NA & \multicolumn{2}{|c|}{ NA } & \multicolumn{2}{|c|}{86.58} & \multicolumn{2}{|c|}{81.28} \\
\hline$S_{1}$ & \multicolumn{2}{|c|}{34.702} & & & 31.272 & \multicolumn{2}{|c|}{-0.080} & \multicolumn{2}{|c|}{1.477} & \multicolumn{2}{|c|}{1.988} \\
\hline$S_{20}$ & \multicolumn{2}{|c|}{-6.892} & & & 1.649 & \multicolumn{2}{|c|}{35.499} & 4.8 & & \multicolumn{2}{|c|}{0.313} \\
\hline \multicolumn{12}{|c|}{ Iran } \\
\hline & \multicolumn{2}{|c|}{ OLS-GQ } & \multicolumn{2}{|c|}{ MD-GQ } & NLS-Beta & $\mathrm{MD}$ & Beta & MD- & B2 & $\mathrm{MD}$ & SCS \\
\hline & Par & SE & Par & SE & Par & Par & SE & Par & SE & Par & SE \\
\hline$\beta_{1} / p$ & 0.888 & 0.004 & 0.922 & 0.011 & 0.714 & 0.698 & 0.005 & 3.059 & 0.602 & -0.803 & 0.257 \\
\hline$\beta_{2} / q$ & -1.030 & 0.018 & -1.190 & 0.022 & 0.962 & 0.947 & 0.003 & 2.258 & 0.377 & 0.732 & 0.026 \\
\hline$\beta_{3} / a$ & 0.212 & 0.008 & 0.135 & 0.005 & 0.517 & 0.488 & 0.011 & 1.426 & 0.149 & 2.128 & 0.253 \\
\hline$\mu$ & NA & NA & 195.99 & 1.69 & 197.73 & 200.41 & 2.09 & 196.96 & 1.80 & 197.27 & 1.78 \\
\hline Gini & 0.383 & 0.000 & 0.385 & 0.004 & 0.384 & 0.391 & 0.005 & 0.381 & 0.004 & 0.383 & 0.004 \\
\hline$H$ & 0.004 & NA & 0.023 & 0.001 & 0.011 & 0.016 & 0.001 & 0.018 & 0.001 & 0.020 & 0.001 \\
\hline$y_{\min }$ & & & 30 & & 0 & & & $c$ & & 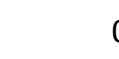 & \\
\hline$y_{\max }$ & 189 & & 271 & & & & & $\alpha$ & & $\alpha$ & \\
\hline$J$-Stat & 913 & & 187 & & NA & & & 33. & & 56 & \\
\hline$S_{1}$ & & & -3.8 & & 8.505 & 0.1 & & -0.4 & & -3.2 & \\
\hline$S_{20}$ & -0. & & -0.1 & & 0.828 & & & -2.4 & & -1. & \\
\hline
\end{tabular}

Notes: OLS-GQ parameters and standard errors are obtained by application of OLS to (7.1). In this case we do not estimate $\mu$. No estimates are reported for MD-GQ for Kenya because its second moment is infinite. MD-GQ, MD-GB2 and MD-SCS estimates are obtained by applying the minimum distance method (3.17). Their standard errors use (3.18). NLS-Beta is obtained by application of nonlinear least squares to the beta GLC, but no NLS standard errors are reported since such standard errors do not have a sound statistical basis. MD-Beta is obtained by application of the minimum distance method, but, since the second order moment often turns out to be infinite, we used $c_{N}=0.99999$ (instead of 1 ) to make estimation feasible, and to enable us to report some numbers. This alternative is not ideal, but an alternative inference method is not available. A $J$-statistic $J=T\left(\tilde{\mathbf{y}}_{L}-\mathbf{G}(\mathbf{c} ; \hat{\phi})\right)^{\prime} \hat{\mathbf{\Omega}}_{L, 22}^{-1}\left(\tilde{\mathbf{y}}_{L}-\mathbf{G}(\mathbf{c} ; \hat{\phi})\right)$ is computed for the minimum distance cases (except for the Beta GLC where it suffered from heavy-tail issues), and also for OLS-GQ and NLS-Beta. In these latter 2 cases we used the estimated parameters to compute a weight matrix as described by MD theory. The critical value for the $J$-statistic at a 0.05 level of significance is $\chi_{16}^{2}=26.3 . S_{1}$ and $S_{20}$ are the percentage errors in prediction of the first and last groups' income. 
Supplementary Web-Based Appendices for

Minimum Distance Estimation of Parametric

Lorenz Curves based on Grouped Data

Gholamreza Hajargasht

Swinburne Business School

William E. Griffiths

University of Melbourne 
Appendix A Derivation of $\Omega_{L, 12}=\operatorname{cov}\left(\sqrt{T} \tilde{\mathbf{z}}, \sqrt{T} \tilde{\mathbf{y}}_{L}\right)$

To our knowledge $\operatorname{cov}\left(\sqrt{T} \tilde{\mathbf{z}}, \sqrt{T} \tilde{\mathbf{y}}_{L}\right)$ has not been provided in the literature. We follow Cowell and Victoria-Feser (2007) and denote the influence function corresponding to $\tilde{z}_{i}$ by $\operatorname{IF}\left(y ; z_{i}\right)$ and that corresponding to $\tilde{y}_{L, j}$ by $I F\left(y ; m_{i}\right)$, we have

$$
\operatorname{cov}\left(\sqrt{T} \tilde{z}_{i}, \sqrt{T} \tilde{y}_{L, j}\right)=\int I F\left(y ; z_{i}\right) \operatorname{IF}\left(y ; m_{i}\right) f(y) d y
$$

It has also been shown that (see Cowell and Victoria-Feser 2007)

$$
\begin{gathered}
I F\left(y ; z_{i}\right)=\frac{c_{i}-g\left(y \leq z_{i}\right)}{f\left(z_{i}\right)} \\
I F\left(y ; m_{i}\right)=c_{i} z_{i}-m_{i}+g\left(y \leq z_{i}\right)\left(y-z_{i}\right)
\end{gathered}
$$

where $m_{i}=G\left(c_{i}\right)$ and $g\left(y \leq z_{i}\right)=1$ when $y \leq z_{i}$, and is 0 otherwise. Using the above facts, we have, for $i=1, \ldots, N-1$ and $j=1, \ldots, N$,

(a) if $i \leq j$

$\operatorname{cov}\left(\sqrt{T} \tilde{z}_{i}, \sqrt{T} \tilde{y}_{L, j}\right)=\int \frac{c_{i}-g\left(y \leq z_{i}\right)}{f\left(z_{i}\right)}\left(c_{j} z_{j}-m_{j}+g\left(y \leq z_{j}\right)\left(y-z_{j}\right)\right) f(y) d y=\frac{c_{i}\left(m_{j}-z_{j} c_{j}\right)-\left(m_{i}-z_{j} c_{i}\right)}{f\left(z_{i}\right)}$

(b) if $i \geq j$

$$
\operatorname{cov}\left(\sqrt{T} \tilde{z}_{i}, \sqrt{T} \tilde{y}_{L, j}\right)=\int \frac{c_{i}-g\left(y \leq z_{i}\right)}{f\left(z_{i}\right)}\left(c_{j} z_{j}-m_{j}+g\left(y \leq z_{j}\right)\left(y-z_{j}\right)\right) f(y) d y=\frac{\left(c_{i}-1\right)\left(m_{j}-z_{j} c_{j}\right)}{f\left(z_{i}\right)}
$$

\section{Appendix B}

Asymptotic distribution for $\mathbf{H}_{m}$

$$
\sqrt{T} \mathbf{H}_{m}=\sqrt{T}\left[\begin{array}{c}
\tilde{\mathbf{c}}-\mathbf{F}(\mathbf{z} ; \phi) \\
\tilde{\mathbf{y}}_{m}-\mathbf{m}(\mathbf{z} ; \phi)
\end{array}\right] \rightarrow N\left(\mathbf{0}, \boldsymbol{\Omega}_{m}\right)
$$




$$
\boldsymbol{\Omega}_{m}=\left[\begin{array}{ccc:ccc}
F_{1}\left(1-F_{1}\right) & \cdots & F_{1}\left(1-F_{N-1}\right) & m_{1}-m_{1} F_{1} & \cdots & m_{1}-F_{1} m_{N} \\
\vdots & \ddots & \vdots & \vdots & \ddots & \vdots \\
F_{1}\left(1-F_{N-1}\right) & \cdots & F_{N-1}\left(1-F_{N-1}\right) & m_{1}-m_{1} F_{N-1} & \cdots & m_{N-1}-F_{N-1} m_{N} \\
m_{1}-m_{1} F_{1} & \cdots & m_{1}-m_{1} F_{N-1} & m_{1}^{(2)}-m_{1}^{2} & \cdots & m_{1}^{(2)}-m_{1} m_{N} \\
\vdots & \ddots & \vdots & \vdots & \ddots & \vdots \\
m_{1}-F_{1} m_{N} & \cdots & m_{N-1}-F_{N-1} m_{N} & m_{1}^{(2)}-m_{1} m_{N} & \cdots & m_{N}^{(2)}-m_{N}^{2}
\end{array}\right]
$$

This follows from the Lindeberg-Levy central limit theorem and is well known in the literature for the separate components $\sqrt{T}(\tilde{\mathbf{c}}-\mathbf{F}(\mathbf{z} ; \phi))$ and $\sqrt{T}\left(\tilde{\mathbf{y}}_{m}-\mathbf{m}(\mathbf{z} ; \phi)\right)$. Here we again use influence functions to derive the complete set of elements in the covariance matrix $\boldsymbol{\Omega}_{m}$.

$$
\begin{gathered}
\operatorname{var}\left(\sqrt{T} \tilde{c}_{i}\right)=\int I F\left(y ; F_{i}\right)^{2} f(y) d y=\int\left[g\left(y \leq z_{i}\right)-F_{i}\right]^{2} f(y) d y=F_{i}-F_{i}^{2}-F_{i}^{2}+F_{i}^{2}=F_{i}\left(1-F_{i}\right) \\
\operatorname{var}\left(\sqrt{T} \tilde{y}_{m, i}\right)=\int I F\left(y ; m_{i}\right)^{2} f(y) d y=\int\left[y g\left(y \leq z_{i}\right)-m_{i}\right]^{2} f(y) d y=m_{i}^{(2)}-m_{i}^{2}-m_{i}^{2}+m_{i}^{2}=m_{i}^{(2)}-m_{i}^{2} \\
\operatorname{cov}\left(\sqrt{T} \tilde{c}_{i}, \sqrt{T} \tilde{y}_{m, i}\right)=\int I F\left(y ; F_{i}\right) I F\left(y ; m_{i}\right) f(y) d y=\int\left[g\left(y \leq z_{i}\right)-F_{i}\right]\left[y g\left(y \leq z_{i}\right)-m_{i}\right] f(y) d y \\
=m_{i}-F_{i} m_{i}-F_{i} m_{i}+F_{i} m_{i}=m_{i}\left(1-F_{i}\right)
\end{gathered}
$$

Also, for every $i$ and $j$ such that $i \leq j$

$$
\begin{aligned}
& \operatorname{cov}\left(\sqrt{T} \tilde{c}_{i}, \sqrt{T} \tilde{c}_{j}\right)=\int I F\left(y ; F_{i}\right) \operatorname{IF}\left(y ; F_{j}\right) f(y) d y=\int\left[g\left(y \leq z_{i}\right)-F_{i}\right]\left[g\left(y \leq z_{j}\right)-F_{j}\right] f(y) d y \\
& =F_{i}-F_{i} F_{j}-F_{i} F_{j}+F_{i} F_{j}=F_{i}\left(1-F_{j}\right) \\
& \operatorname{cov}\left(\sqrt{T} \tilde{y}_{m, i}, \sqrt{T} \tilde{y}_{m, j}\right)=\int I F\left(y ; m_{i}\right) \operatorname{IF}\left(y ; m_{j}\right) f(y) d y=\int\left[y g\left(y \leq z_{i}\right)-m_{i}\right]\left[y g\left(y \leq z_{j}\right)-m_{j}\right] f(y) d y \\
& =m_{i}^{(2)}-m_{i} m_{j}-m_{i} m_{j}+m_{i} m_{j}=m_{i}^{(2)}-m_{i} m_{j} \\
& \operatorname{cov}\left(\sqrt{T} \tilde{c}_{i}, \sqrt{T} \tilde{y}_{m, j}\right)=\int \operatorname{IF}\left(y ; F_{i}\right) \operatorname{IF}\left(y ; m_{j}\right) f(y) d y=\int\left[g\left(y \leq z_{i}\right)-F_{i}\right]\left[y g\left(y \leq z_{j}\right)-m_{j}\right] f(y) d y \\
& =m_{i}-F_{i} m_{j}-F_{i} m_{j}+F_{i} m_{j}=m_{i}-F_{i} m_{j}
\end{aligned}
$$

\section{Derivation of the optimal weight matrix $\mathrm{W}_{m}$}

Obtaining the weight matrix in (4.4) requires inverting $\Omega_{m}$. Hajargasht et al. (2012) have derived the weight matrix for a situation where population proportions and income are defined non-cumulatively. To use their result to derive the weight matrix for the cumulative population 
proportions and income considered here, we begin by defining the $(N \times N)$ matrix $\mathbf{A}_{N}$ and the $[(2 N-1) \times(2 N-1)]$ matrix $\mathbf{B}$ as follows

$$
\mathbf{A}_{N}=\left[\begin{array}{cccc}
1 & & & \\
-1 & 1 & & \\
& \ddots & \ddots & \\
& & -1 & 1
\end{array}\right] \quad \mathbf{B}=\left[\begin{array}{cc}
\mathbf{A}_{N-1} & \mathbf{0}_{N-1, N} \\
\mathbf{0}_{N, N-1} & \mathbf{A}_{N}
\end{array}\right]
$$

Let $\mathbf{H}^{*}(\boldsymbol{\theta})=\mathbf{B} \mathbf{H}_{m}(\boldsymbol{\theta})$. The relationship between the asymptotic covariance matrices for $\mathbf{H}^{*}(\boldsymbol{\theta})$ and $\mathbf{H}_{m}(\boldsymbol{\theta})$ is $\operatorname{var}\left[T^{1 / 2} \mathbf{H}^{*}(\boldsymbol{\theta})\right]=\mathbf{B} \operatorname{var}\left[T^{1 / 2} \mathbf{H}_{m}(\boldsymbol{\theta})\right] \mathbf{B}^{\prime}$ from which we obtain

$$
\operatorname{var}\left[T^{1 / 2} \mathbf{H}_{m}(\boldsymbol{\theta})\right]=\mathbf{B}^{-1} \operatorname{var}\left[T^{1 / 2} \mathbf{H}^{*}(\boldsymbol{\theta})\right] \mathbf{B}^{\prime-1}
$$

The required weight matrix is

$$
\mathbf{W}_{m}=\left(\operatorname{var}\left[T^{1 / 2} \mathbf{H}_{m}(\boldsymbol{\theta})\right]\right)^{-1}=\mathbf{B}^{\prime}\left(\operatorname{var}\left[T^{1 / 2} \mathbf{H}^{*}(\boldsymbol{\theta})\right]\right)^{-1} \mathbf{B}
$$

In Hajargasht et al. (2012) it is shown that

$$
\left(\operatorname{var}\left[T^{1 / 2} \mathbf{H}^{*}(\boldsymbol{\theta})\right]\right)^{-1}=\left[\begin{array}{ccc}
D\left(\boldsymbol{\mu}_{-N}^{(2)} / \mathbf{v}_{-N}\right)+\left(\mu_{N}^{(2)} / \nu_{N}\right) \mathbf{j}_{N-1} \mathbf{j}_{N-1}^{\prime} & -D\left(\boldsymbol{\mu}_{-N} / \mathbf{v}_{-N}\right) & \left(\mu_{N} / v_{N}\right) \mathbf{j}_{N-1} \\
-D\left(\boldsymbol{\mu}_{-N} / \mathbf{v}_{-N}\right) & D\left(\boldsymbol{\kappa}_{-N} / \mathbf{v}_{-N}\right) & \mathbf{0} \\
\left(\mu_{N} / v_{N}\right) \mathbf{j}_{N-1}^{\prime} & \mathbf{0} & \kappa_{N} / v_{N}
\end{array}\right]
$$

where $\quad \boldsymbol{\mu}_{-N}^{\prime}=\left(\mu_{1}, \mu_{2}, \ldots, \mu_{N-1}\right), \quad \boldsymbol{\mu}_{-N}^{(2)}=\left(\mu_{1}^{(2)}, \mu_{2}^{(2)}, \ldots, \mu_{N}^{(2)}\right), \quad \boldsymbol{\kappa}_{-N}^{\prime}=\left(\kappa_{1}, \kappa_{2}, \ldots, \kappa_{N-1}\right), \quad$ and $\mathbf{v}_{-N}^{\prime}=\left(v_{1}, v_{2}, \ldots, v_{N-1}\right)$. Using (B.1) and (B.4) to carry out the matrix multiplication in (B.3) yields the weight matrix $\mathbf{W}_{m}=\left[\begin{array}{ll}\mathbf{W}_{m, 11} & \mathbf{W}_{m, 12} \\ \mathbf{W}_{m, 12}^{\prime} & \mathbf{W}_{m, 22}\end{array}\right]$.

To specify the elements in each of the blocks of $\mathbf{W}_{m}$, we define

$$
\begin{gathered}
\kappa_{i}=\kappa_{i}(\boldsymbol{\theta})=F\left(z_{i} ; \phi\right)-F\left(z_{i-1} ; \phi\right), \\
\mu_{i}=\mu_{i}(\boldsymbol{\theta})=m\left(z_{i} ; \phi\right)-m\left(z_{i-1} ; \phi\right), \\
\mu_{i}^{(2)}=\mu_{i}^{(2)}(\boldsymbol{\theta})=m^{(2)}\left(z_{i} ; \phi\right)-m^{(2)}\left(z_{i-1} ; \phi\right)
\end{gathered}
$$




$$
v_{i}=\kappa_{i} \mu_{i}^{(2)}-\mu_{i}^{2}
$$

Each of the blocks $\mathbf{W}_{m, i j}$ is tri-diagonal, with the following elements

$$
\begin{aligned}
& \mathbf{W}_{m, 11}=\left[\begin{array}{lll}
\frac{\mu_{2}^{(2)}}{v_{2}}+\frac{\mu_{1}^{(2)}}{v_{1}}-\mu_{2}^{(2)} / v_{2} & \\
-\mu_{2}^{(2)} / v_{2} & -\mu_{N-1}^{(2)} / v_{N-1} \\
& -\mu_{N-1}^{(2)} / v_{N-1} & \frac{\mu_{N}^{(2)}}{v_{N}}+\frac{\mu_{N-1}^{(2)}}{v_{N-1}}
\end{array}\right] \\
& \mathbf{W}_{m, 12}=\left[\begin{array}{llll}
\frac{-\mu_{2}}{v_{2}}-\frac{\mu_{1}}{v_{1}} & \mu_{2} / v_{2} & & \\
\mu_{2} / v_{2} \\
& \mu_{N-1} / v_{N-1} & \frac{-\mu_{N}}{v_{N}}-\frac{\mu_{N-1}}{v_{N-1}} & \mu_{N} / v_{N}
\end{array}\right] \\
& \mathbf{W}_{m, 22}=\left[\begin{array}{lll}
\frac{\kappa_{1}}{v_{1}}+\frac{\kappa_{2}}{v_{2}} & -\kappa_{1} / v_{1} & \\
-\kappa_{1} / v_{1} & & \\
& \frac{\kappa_{N-1}}{v_{N-1}}+\frac{\kappa_{N}}{v_{N}} & -\kappa_{N-1} / v_{N-1} \\
& -\kappa_{N-1} / v_{N-1} & -\kappa_{N} / v_{N}
\end{array}\right]
\end{aligned}
$$

\section{Appendix C1}

\section{Proof of Proposition 1}

Proof: (i) can be shown by direct multiplication after recognising that $c_{i}=F\left(z_{i}\right)$ and that this implies $m\left(z_{i}\right)=G\left(c_{i}\right)$. To prove (ii) note that $\mathbf{W}_{L}=\mathbf{\Omega}_{L}^{-1}=\left(\mathbf{A} \boldsymbol{\Omega}_{m} \mathbf{A}^{\prime}\right)^{-1}=\mathbf{A}^{\prime-1} \mathbf{W}_{m} \mathbf{A}^{-1}$. The particular form of $\mathbf{A}^{-1}$ can be checked by showing that $\mathbf{A} \mathbf{A}^{-1}=\mathbf{I}$. The equality of the lower diagonal blocks of $\mathbf{W}_{L}$ and $\mathbf{W}_{m}$ can be checked by multiplication. The first equality in (iii) comes from (i). To prove the second equality in (iii), we first note that

$$
\mathbf{B}^{\prime}\left(\mathbf{B B}^{\prime}\right)^{-1} \mathbf{B}=\mathbf{I}-\mathbf{C}\left(\mathbf{C}^{\prime} \mathbf{C}\right)^{-1} \mathbf{C}^{\prime}
$$


from which it follows that

$$
\left[\mathbf{I}-\mathbf{C}\left(\mathbf{C}^{\prime} \mathbf{W}_{m} \mathbf{C}\right)^{-1} \mathbf{C}^{\prime} \mathbf{W}_{m}\right] \mathbf{B}^{\prime}(\mathbf{B B})^{\prime-1} \mathbf{B}=\left[\mathbf{I}-\mathbf{C}\left(\mathbf{C}^{\prime} \mathbf{W}_{m} \mathbf{C}\right)^{-1} \mathbf{C}^{\prime} \mathbf{W}_{m}\right]
$$

Then, postmultiplying the right-hand side of (iii) by $\mathbf{B} \boldsymbol{\Omega}_{m} \mathbf{B}^{\prime}$ yields

$$
\begin{aligned}
& (\mathbf{B B})^{-1} \mathbf{B} \mathbf{W}_{m}\left[\mathbf{I}-\mathbf{C}\left(\mathbf{C}^{\prime} \mathbf{W}_{m} \mathbf{C}\right)^{-1} \mathbf{C}^{\prime} \mathbf{W}_{m}\right] \mathbf{B}^{\prime}\left(\mathbf{B} \mathbf{B}^{\prime}\right)^{-1} \times\left[\mathbf{B} \boldsymbol{\Omega}_{m} \mathbf{B}^{\prime}\right] \\
= & \left(\mathbf{B B}^{\prime}\right)^{-1} \mathbf{B} \mathbf{W}_{m}\left[\mathbf{I}-\mathbf{C}\left(\mathbf{C}^{\prime} \mathbf{W}_{m} \mathbf{C}\right)^{-1} \mathbf{C}^{\prime} \mathbf{W}_{m}\right] \mathbf{\Omega}_{m} \mathbf{B}^{\prime} \\
= & \left(\mathbf{B B}^{\prime}\right)^{-1} \mathbf{B} \mathbf{W}_{m}\left[\mathbf{\Omega}_{m} \mathbf{B}^{\prime}-\mathbf{C}\left(\mathbf{C}^{\prime} \mathbf{W}_{m} \mathbf{C}\right)^{-1} \mathbf{C}^{\prime} \mathbf{B}^{\prime}\right] \quad \text { since } \mathbf{W}_{m} \mathbf{\Omega}_{m}=\mathbf{I} \\
= & (\mathbf{B B})^{-1} \mathbf{B} \mathbf{B}^{\prime} \quad \text { since } \mathbf{C}^{\prime} \mathbf{B}^{\prime}=\mathbf{0} \\
= & \mathbf{I}
\end{aligned}
$$

Result (ii) in this proposition provides a closed form for the weight matrix for DGP1 when both quantile and Lorenz equations are considered. The result in (iii) provides a tractable formula for the weight matrix when only the Lorenz equations are considered (see equation (3.17)). Its tractability comes from the facts that $\mathbf{B B}^{\prime}$ is diagonal, $\mathbf{C}^{\prime} \mathbf{W}_{m} \mathbf{C}$ is tri-diagonal and there are special formulae for inverting such matrices.

\section{Proof of Proposition 2}

(i) Using Lemma 2, and the matrix A defined in Proposition 1, it can be shown that

$$
\left[\begin{array}{ll}
\frac{\partial \mathbf{F}^{\prime-1}}{\partial \boldsymbol{\phi}} & \frac{\partial \mathbf{G}^{\prime}}{\partial \phi}
\end{array}\right]=\left[\begin{array}{ll}
\frac{\partial \mathbf{F}^{\prime}}{\partial \phi} & \frac{\partial \mathbf{m}^{\prime}}{\partial \phi}
\end{array}\right] \mathbf{A}^{\prime}
$$

From this result and that $\mathbf{W}_{L}=\mathbf{A}^{\prime-1} \mathbf{W}_{m} \mathbf{A}^{-1}$ from Proposition 1, we have

$$
\begin{aligned}
\operatorname{cov}\left(\hat{\boldsymbol{\phi}}_{L}\right) & =\frac{1}{T}\left(\left[\begin{array}{ll}
\frac{\partial \mathbf{F}^{\prime-1}}{\partial \phi} & \frac{\partial \mathbf{G}^{\prime}}{\partial \phi}
\end{array}\right] \mathbf{W}_{L}(\phi)\left[\begin{array}{l}
\partial \mathbf{F}^{-1} / \partial \phi^{\prime} \\
\partial \mathbf{G} / \partial \phi^{\prime}
\end{array}\right]\right)^{-1}=\frac{1}{T}\left(\left[\begin{array}{ll}
\frac{\partial \mathbf{F}^{\prime}}{\partial \phi} & \frac{\partial \mathbf{m}^{\prime}}{\partial \phi}
\end{array}\right] \mathbf{A}^{\prime} \mathbf{A}^{\prime-1} \mathbf{W}_{m} \mathbf{A}^{-1} \mathbf{A}\left[\begin{array}{l}
\partial \mathbf{F} / \partial \boldsymbol{\phi}^{\prime} \\
\partial \mathbf{m} / \partial \boldsymbol{\phi}^{\prime}
\end{array}\right]\right)^{-1} \\
& =\frac{1}{T}\left(\left[\begin{array}{ll}
\frac{\partial \mathbf{F}^{\prime}}{\partial \phi} & \frac{\partial \mathbf{m}^{\prime}}{\partial \phi}
\end{array}\right] \mathbf{W}_{m}\left[\begin{array}{l}
\partial \mathbf{F} / \partial \phi^{\prime} \\
\partial \mathbf{m} / \partial \phi^{\prime}
\end{array}\right]\right)^{-1}=\operatorname{cov}\left(\hat{\phi}_{m}\right)
\end{aligned}
$$

(ii) First note that

$$
\operatorname{cov}\left(\hat{\phi}_{L}\right)=\frac{1}{T}\left(\frac{\partial \mathbf{G}^{\prime}}{\partial \phi} \mathbf{\Omega}_{L, 22}^{-1}(\phi) \frac{\partial \mathbf{G}}{\partial \phi^{\prime}}\right)^{-1}
$$


Recall that $\partial \mathbf{H}_{m}^{\prime} / \partial \phi=\left(\partial \mathbf{F}^{\prime} / \partial \phi \quad \partial \mathbf{m}^{\prime} / \partial \phi\right)$. From Proposition 1 and Lemma 2(iii), and recognising that $\partial G_{N} / \partial \phi=\partial m_{N} / \partial \phi$, we can write $\partial \mathbf{G} / \partial \phi^{\prime}=\mathbf{B}\left(\partial \mathbf{H}_{m} / \partial \phi^{\prime}\right)$, and

$$
\begin{aligned}
\operatorname{cov}\left(\hat{\phi}_{L}\right) & =\frac{1}{T}\left(\frac{\partial \mathbf{H}_{m}^{\prime}}{\partial \phi} \mathbf{B}^{\prime}\left(\mathbf{B} \mathbf{B}^{\prime}\right)^{-1} \mathbf{B}\left[\mathbf{W}_{m}-\mathbf{W}_{m} \mathbf{C}\left(\mathbf{C}^{\prime} \mathbf{W}_{m} \mathbf{C}\right)^{-1} \mathbf{C}^{\prime} \mathbf{W}_{m}\right] \mathbf{B}^{\prime}\left(\mathbf{B} \mathbf{B}^{\prime}\right)^{-1} \mathbf{B} \frac{\partial \mathbf{H}_{m}}{\partial \phi^{\prime}}\right)^{-1} \\
& =\frac{1}{T}\left(\frac{\partial \mathbf{H}_{m}^{\prime}}{\partial \phi}\left[\mathbf{W}_{m}-\mathbf{W}_{m} \mathbf{C}\left(\mathbf{C}^{\prime} \mathbf{W}_{m} \mathbf{C}\right)^{-1} \mathbf{C}^{\prime} \mathbf{W}_{m}\right] \frac{\partial \mathbf{H}_{m}}{\partial \phi^{\prime}}\right)^{-1}
\end{aligned}
$$

The last equality comes from a result established in Proposition 1, namely,

$$
\left[\mathbf{I}-\mathbf{C}\left(\mathbf{C}^{\prime} \mathbf{W}_{m} \mathbf{C}\right)^{-1} \mathbf{C}^{\prime} \mathbf{W}_{m}\right] \mathbf{B}^{\prime}(\mathbf{B B})^{\prime-1} \mathbf{B}=\left[\mathbf{I}-\mathbf{C}\left(\mathbf{C}^{\prime} \mathbf{W}_{m} \mathbf{C}\right)^{-1} \mathbf{C}^{\prime} \mathbf{W}_{m}\right]
$$

Now,

$$
\operatorname{cov}\left(\begin{array}{c}
\hat{\mathbf{z}} \\
\hat{\boldsymbol{\phi}}_{m}
\end{array}\right)=\frac{1}{T}\left(\begin{array}{cc}
\left(\partial \mathbf{H}_{m}^{\prime} / \partial \mathbf{z}\right) \mathbf{W}_{m}\left(\partial \mathbf{H}_{m} / \partial \mathbf{z}^{\prime}\right) & \left(\partial \mathbf{H}_{m}^{\prime} / \partial \mathbf{z}\right) \mathbf{W}_{m}\left(\partial \mathbf{H}_{m} / \partial \phi^{\prime}\right) \\
\left(\partial \mathbf{H}_{m}^{\prime} / \partial \phi\right) \mathbf{W}_{m}\left(\partial \mathbf{H}_{m} / \partial \mathbf{z}^{\prime}\right) & \left(\partial \mathbf{H}_{m}^{\prime} / \partial \phi\right) \mathbf{W}_{m}\left(\partial \mathbf{H}_{m} / \partial \phi^{\prime}\right)
\end{array}\right)^{-1}
$$

Using the formula for the inverse of a partitioned matrix, we have

$$
\operatorname{cov}\left(\hat{\phi}_{m}\right)=\frac{1}{T}\left(\frac{\partial \mathbf{H}_{m}^{\prime}}{\partial \phi} \mathbf{W}_{m} \frac{\partial \mathbf{H}_{m}}{\partial \phi^{\prime}}-\frac{\partial \mathbf{H}_{m}^{\prime}}{\partial \phi} \mathbf{W}_{m} \frac{\partial \mathbf{H}_{m}}{\partial \mathbf{z}^{\prime}}\left(\frac{\partial \mathbf{H}_{m}^{\prime}}{\partial \mathbf{z}} \mathbf{W}_{m} \frac{\partial \mathbf{H}_{m}}{\partial \mathbf{z}^{\prime}}\right)^{-1} \frac{\partial \mathbf{H}_{m}^{\prime}}{\partial \mathbf{z}} \mathbf{W}_{m} \frac{\partial \mathbf{H}_{m}}{\partial \phi^{\prime}}\right)^{-1}
$$

Now,

$$
\frac{\partial \mathbf{H}_{m}^{\prime}}{\partial \mathbf{z}}=\left[\begin{array}{lll}
D(\mathbf{f}(\mathbf{z})) & D(\mathbf{z . f}(\mathbf{z})) & \mathbf{0}
\end{array}\right]=D(\mathbf{f}(\mathbf{z})) \mathbf{C}^{\prime}
$$

and thus,

$$
\begin{aligned}
\operatorname{cov}\left(\hat{\phi}_{m}\right) & =\frac{1}{T}\left(\frac{\partial \mathbf{H}_{m}^{\prime}}{\partial \phi}\left\{\mathbf{W}_{m}-\mathbf{W}_{m} \mathbf{C D}(\mathbf{f}(\mathbf{z}))\left[D(\mathbf{f}(\mathbf{z})) \mathbf{C}^{\prime} \mathbf{W}_{m} \mathbf{C D}(\mathbf{f}(\mathbf{z}))\right]^{-1} D(\mathbf{f}(\mathbf{z})) \mathbf{C}^{\prime} \mathbf{W}_{m}\right\} \frac{\partial \mathbf{H}_{m}}{\partial \phi^{\prime}}\right)^{-1} \\
& =\frac{1}{T}\left(\frac{\partial \mathbf{H}_{m}^{\prime}}{\partial \phi}\left[\mathbf{W}_{m}-\mathbf{W}_{m} \mathbf{C}\left(\mathbf{C}^{\prime} \mathbf{W}_{m} \mathbf{C}\right)^{-1} \mathbf{C}^{\prime} \mathbf{W}_{m}\right] \frac{\partial \mathbf{H}_{m}}{\partial \phi^{\prime}}\right)^{-1}
\end{aligned}
$$

which proves the proposition. 


\section{Appendix D Inference for standard Lorenz curves}

The above results can be used to provide inference based on the standard Lorenz curve. We can define the moment conditions for the standard Lorenz curve by

$$
\text { DGP1: } \underset{(2 N-2) \times 1}{\mathbf{P}_{L}}=\left[\begin{array}{c}
\tilde{z}_{1}-F^{-1}\left(c_{1}\right) \\
\vdots \\
\tilde{z}_{N-1}-F^{-1}\left(c_{N-1}\right) \\
\tilde{y}_{L, 1} / \tilde{y}_{L, N}-G_{1} / m_{N} \\
\vdots \\
\tilde{y}_{L, N-1} / \tilde{y}_{L, N}-G_{N-1} / m_{N}
\end{array}\right]
$$$$
\operatorname{DGP} 2: \underset{(2 N-2) \times 1}{\mathbf{P}_{m}}=\left[\begin{array}{c}
c_{1}-F_{1} \\
\vdots \\
c_{N-1}-F_{N-1} \\
\tilde{y}_{m, 1} / \tilde{y}_{m, N}-m_{1} / m_{N} \\
\vdots \\
\tilde{y}_{m, N-1} / \tilde{y}_{m, N}-m_{N-1} / m_{N}
\end{array}\right]
$$

For DGP1, let

$$
\boldsymbol{\eta}=\left[\begin{array}{c}
\tilde{z}_{1} \\
\vdots \\
\tilde{z}_{N-1} \\
\tilde{y}_{L, 1} / \tilde{y}_{L, N} \\
\vdots \\
\tilde{y}_{L, N-1} / \tilde{y}_{L, N}
\end{array}\right] \text { and } \tilde{\boldsymbol{\eta}}=\left[\begin{array}{c}
\tilde{z}_{1} \\
\vdots \\
\tilde{z}_{N-1} \\
\tilde{y}_{L, 1} \\
\vdots \\
\tilde{y}_{L, N}
\end{array}\right] \quad \text { so that } \quad \boldsymbol{\eta}=\left[\begin{array}{c}
\tilde{\eta}_{1} \\
\vdots \\
\tilde{\eta}_{N-1} \\
\tilde{\eta}_{N} / \tilde{\eta}_{2 N-1} \\
\vdots \\
\tilde{\eta}_{2 N-2} / \tilde{\eta}_{2 N-1}
\end{array}\right]
$$

Then, using the delta method, the covariance matrix of the limiting distribution can be written as

$$
\operatorname{cov}\left[T^{1 / 2} \mathbf{P}_{L}\right]=\operatorname{plim}\left(\frac{\partial \boldsymbol{\eta}}{\partial \tilde{\boldsymbol{\eta}}^{\prime}}\right) \operatorname{cov}\left(T^{1 / 2} \tilde{\boldsymbol{\eta}}\right) \operatorname{plim}\left(\frac{\partial \mathbf{\eta}^{\prime}}{\partial \tilde{\boldsymbol{\eta}}}\right)
$$

Differentiating and taking probability limits, yields

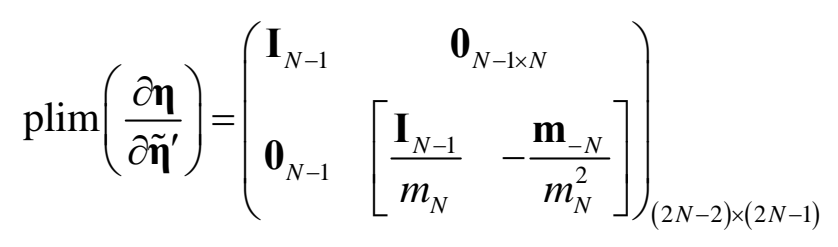

A similar result can be obtained for DGP2. Nothing that $\operatorname{cov}\left(T^{1 / 2} \tilde{\boldsymbol{\eta}}\right)$ is given in (3.7), the required asymptotic variance can be computed from (D.2) and (D.3). For the weight matrix in minimum distance estimation $\operatorname{cov}\left[T^{1 / 2} \mathbf{P}_{L}\right]$ needs to be inverted. 


\section{Appendix E Monte Carlo experiments}

Here we perform a Monte Carlo experiment to address the following questions: (i) Do the estimators perform well? (ii) Does the method of grouping matter? (iii) How do the various estimators compare with each other and with some traditional Lorenz curve estimation methods? (iv) How do the estimators perform in finite samples?

With the exception of one experiment, the data for all simulations were obtained by drawing 10,000 observations from a GB2 distribution with $b=100, p=1, q=1.5$ and $a=1.5$, implying a heavy-tailed Singh-Maddala distribution with a Gini coefficient of $0.53 .{ }^{1}$ A heavytailed case was considered because inference in such cases can be more challenging.

In the first experiment we investigate the performance of covariance matrix estimators for the GLC ordinates without making any distributional assumptions. The generated 10,000 observations were used to create 5 groups based on the two grouping methods. For DGP1, the data were grouped by dividing the simulated sample into 5 equally-sized groups with $\mathbf{c}^{\prime}=(0.2,0.4, \ldots, 0.8)$; then the associated $\tilde{y}_{i}$ s and $\tilde{z}_{i}$ s were computed. For DGP2, the groups bounds $\left(z_{i}{ }^{\prime} s\right)$ were set according to the theoretical quantiles corresponding to $\mathbf{c}$, and the associated $\tilde{y}_{i}$ and $\tilde{c}_{i}$ were computed. We restricted the number of groups to 5 in this case for ease of presentation of the results. For later experiments we used 20 groups with cumulative proportions $\mathbf{c}^{\prime}=(0.05,0.1, \ldots, 0.95)$. The two covariance matrices being considered are $\boldsymbol{\Omega}_{L, 22}$ and $\boldsymbol{\Omega}_{m, 22}$ given in (3.4) and (4.4), respectively. In (3.4), $\tilde{y}_{L, i}$ is used to estimate $G\left(c_{i}\right)$; when the grouping is made, we also compute $\tilde{y}_{L, i}^{(2)}=T^{-1} \sum_{t=1}^{T} y_{t}^{2} g_{i}\left(y_{t}\right)$ which is used to estimate $m_{i}^{(2)}$. In (4.4), $\tilde{y}_{m, i}$ is used to estimate $m_{i}$ and $\tilde{y}_{m, i}^{(2)}$ is used to estimate $m_{i}^{(2)}$.

\footnotetext{
${ }^{1}$ A Singh-Maddala distribution is a special case of the GB2 where $p=1$. $k$-th moment of the GB2 exists if $a q>k$ . Thus, for $a=q=1.5$, moments beyond the second one do not exist.
} 
Table A1 Covariance matrix for $\tilde{y}_{i}$ s using a distribution free approach

\begin{tabular}{|c|c|c|c|c|c|c|c|c|c|c|}
\hline & \multicolumn{5}{|c|}{ Covariance Matrix of Lorenz Ordinates DGP 1} & \multicolumn{5}{|c|}{ Covariance Matrix of Lorenz Ordinates DGP 2} \\
\hline & \multicolumn{5}{|c|}{ Formula (3.4) at Sample Information } & \multicolumn{5}{|c|}{ Formula (4.4) at Sample Information } \\
\hline & $\tilde{y}_{1}$ & $\tilde{y}_{2}$ & $\tilde{y}_{3}$ & $\tilde{y}_{4}$ & $\tilde{y}_{5}$ & $\tilde{y}_{1}$ & $\tilde{y}_{2}$ & $\tilde{y}_{3}$ & $\tilde{y}_{4}$ & $\tilde{y}_{5}$ \\
\hline$\tilde{y}_{1}$ & 377 & 0.822 & 1.249 & 1.722 & 2.598 & 0.578 & 0.296 & -0.182 & -0.973 & -3.193 \\
\hline$\tilde{y}_{2}$ & & & & 6.037 & 9.573 & & 2.912 & & -1.496 & -9.203 \\
\hline$\tilde{y}_{3}$ & & & & & & & & & .731 & -15.267 \\
\hline$\tilde{y}_{4}$ & 1.722 & 6.037 & 13.589 & 26.117 & & -0.973 & -1.496 & 1.731 & 18.314 & -14.072 \\
\hline$\tilde{y}_{5}$ & & 9.57 & 23.10 & 51.97 & & & -9.20 & -15.27 & -14.07 & \\
\hline & \multicolumn{5}{|c|}{ Covariance of Simulated Draws } & \multicolumn{5}{|c|}{ Covariance of Simulated Draws } \\
\hline$\tilde{y}_{1}$ & 0.382 & 0.821 & 1.248 & 1.691 & 2.252 & & & & -1.009 & 192 \\
\hline$\tilde{y}_{2}$ & & & & 5.766 & & & 2.889 & & & -8.402 \\
\hline$\tilde{y}_{3}$ & 248 & 3.993 & 8.011 & 12.813 & & -0.181 & & & 2.035 & -11 \\
\hline$\tilde{y}_{4}$ & 1.691 & 5.766 & 12.813 & 24.883 & & -1.009 & -1.589 & 2.035 & & -12.559 \\
\hline$\tilde{y}_{5}$ & 2.25 & 8.83 & 20.38 & 45.38 & 444.39 & -3.19 & -8.40 & & -12.56 & 444.39 \\
\hline & \multicolumn{5}{|c|}{ Formula (3.4) Computed at True Values } & \multicolumn{5}{|c|}{ Formula (4.4) Computed at True Values } \\
\hline$\tilde{y}_{1}$ & 0.377 & 0.823 & 1.251 & 1.72 & 2.601 & .578 & 0.2 & & & -3.191 \\
\hline$\tilde{y}_{2}$ & & 2.404 & & & & & & & -1.497 & \\
\hline$\tilde{y}_{3}$ & & 4.129 & 8.413 & 13.578 & 23.168 & -0.182 & 1.2 & 7.7 & 1.730 & -15.268 \\
\hline$\tilde{y}_{4}$ & 1.724 & 6.034 & 13.578 & 26.089 & 51.938 & -0.973 & -1.497 & 1.730 & 18.317 & -14.068 \\
\hline$\tilde{y}_{5}$ & 2.60 & 9.57 & 23.17 & 51.94 & & -3.19 & -9.20 & -15.27 & -14.07 & 615.62 \\
\hline & \multicolumn{5}{|c|}{ Weight Matrix Computed at True Values } & \multicolumn{5}{|c|}{ Weight Matrix Computed at True Values } \\
\hline$\tilde{y}_{1}$ & 1335.50 & -777.03 & 220.01 & -23.24 & 0.119 & & & & 10.03 & .26 \\
\hline$\tilde{y}_{2}$ & -777.030 & 806.520 & -389.100 & 67.989 & -0.349 & -15.091 & 42.225 & -7.051 & 3.669 & 0.462 \\
\hline$\tilde{y}_{3}$ & 220.010 & -389.100 & 304.510 & -84.608 & 0.798 & 7.513 & -7.051 & 14.979 & -1.382 & 0.273 \\
\hline$\tilde{y}_{4}$ & -23.244 & 67.989 & -84.608 & 35.151 & -0.740 & 10.029 & 3.669 & -1.382 & 6.594 & 0.223 \\
\hline & 0.119 & -0.349 & 0.798 & -0.740 & 0.200 & 1.263 & 0.462 & 0.273 & 0.223 & 0.188 \\
\hline
\end{tabular}

Table A1 contains the results from 500 replications. Its first panel provides the Monte Carlo averages of the estimates for $\boldsymbol{\Omega}_{L, 22}$ and $\boldsymbol{\Omega}_{m, 22}$. The second panel contains the Monte Carlo covariance matrix of the simulated GLC ordinates. The matrices $\Omega_{L, 22}$ and $\boldsymbol{\Omega}_{m, 22}$ evaluated at the true values of the parameters appear in the $3^{\text {rd }}$ panel, and the inverses of these matrices are in the final panel. The values in the first three panels for each of the DGPs are generally similar confirming the validity of the formulae. There are some large differences between alternative variances of the last group mean where $\tilde{y}_{5}$ is an estimate of the mean $\mu$. 
This appears to be attributable to the heavy-tailed distribution which is close to having an infinite second order moment. A comparison of the DGP1 and DGP2 covariance matrices shows that their variances can be substantially different. The variances for the initial groups are smaller for DGP1, they become larger for the later groups and become equal as expected for the last group where in both cases the mean is being estimated. These results are evident from the formula

$$
\begin{aligned}
& \text { DGP 1: } \quad\left[\boldsymbol{\Omega}_{L, 22}\right]_{i i}=m_{i}^{(2)}-m_{i}^{2}+z_{i}\left(1-c_{i}\right)\left(c_{i} Z_{i}-2 m_{i}\right) \\
& \text { DGP 2: } \quad\left[\boldsymbol{\Omega}_{m, 22}\right]_{i i}=m_{i}^{(2)}-m_{i}^{2}
\end{aligned}
$$

As long as $c_{i} z_{i}<2 m_{i}$ (which happens for the initial groups with typical income distributions) the DGP1 variance is smaller. Also the variances are equal when $c_{i}=1$. We can also see (both theoretically and from the simulation) that the covariances from DGP1 are always positive but for DGP2 they could be positive or negative. Finally, the inverses of the covariance matrices, i.e., the weight matrices for both DGPs, are starkly different from the identity matrix which is often used implicitly in least square estimation.

In the second experiment we consider estimation of the parameters of the GB2 distribution under DGP1 using two scenarios: observations on $\left(c_{i}, \tilde{y}_{L, i}, z_{i}\right)$ and observations on $\left(c_{i}, \tilde{y}_{L, i}\right)$ only. Experimental results from using the minimum distance estimators (3.8) and (3.17) are given in Table A2. Again, 500 Monte Carlo iterations are used. ${ }^{2}$ In addition to the GB2 parameters, we also include the Gini coefficient since it is often a quantity of interest. The left and right panels in the Table display the results for observed and unobserved $\tilde{z}_{i}$, respectively. In each case, the first column provides the average of the estimated parameters over the Monte-Carlo replications. As it can be seen, they are almost identical to the true values, suggesting unbiasedness. The second, third and fourth columns contain, respectively, (i) the

\footnotetext{
${ }^{2}$ In all cases, we conducted repeated minimum distance estimation starting with an identity weight matrix and repeating the process 10 times as explained in Section 3. All estimations were done using code written in MATLAB R2013.
} 
asymptotic variances computed at the true parameter values, (ii) the average of the estimated variances over the replications, and (iii) the Monte Carlo sample variance of the estimated parameters. There are only minor differences between the three sets of variances. The sample variances are slightly larger than their asymptotic counterparts, indicating that there could be some underestimation of the finite sample variation, but, because the differences are not large, overall we can conclude that the estimators perform well in finite samples. We also see that estimation with observed $z_{i}$ 's is more efficient than estimation with unobserved $z_{i}$ 's, but the differences are negligible. At least under the ideal conditions of the Monte Carlo experiment, not knowing the $z_{i}$ 's has little impact.

Table A2 Results from Monte Carlo experiment for DGP1

\begin{tabular}{|c|c|c|c|c|c|c|c|c|c|}
\hline & \multicolumn{4}{|c|}{ With data on $\mathrm{z}$} & \multicolumn{4}{|c|}{ With unknown z } \\
\hline & $\begin{array}{l}\text { True } \\
\text { Par }\end{array}$ & $\begin{array}{l}\text { Average } \\
\text { Est-Par }\end{array}$ & $\begin{array}{l}\text { True } \\
\text { Var }\end{array}$ & $\begin{array}{l}\text { Average } \\
\text { Est-Var }\end{array}$ & $\begin{array}{l}\text { Variance } \\
\text { Est-Par }\end{array}$ & $\begin{array}{l}\text { Average } \\
\text { Est-Par }\end{array}$ & $\begin{array}{l}\text { True } \\
\text { Var }\end{array}$ & $\begin{array}{l}\text { Average } \\
\text { Est-Var }\end{array}$ & $\begin{array}{l}\text { Variance } \\
\text { Est-Par }\end{array}$ \\
\hline$b$ & 100.00 & 100.70 & 32.84 & 34.68 & 36.20 & 100.56 & 34.06 & 36.50 & 37.52 \\
\hline$p$ & 1.0000 & 1.0177 & 0.0122 & 0.0123 & 0.0133 & 1.0155 & 0.0125 & 0.0129 & 0.0136 \\
\hline$q$ & 1.5000 & 1.5338 & 0.0417 & 0.0416 & 0.0464 & 1.5290 & 0.0431 & 0.0440 & 0.0479 \\
\hline$a$ & 1.5000 & 1.4941 & 0.0142 & 0.0133 & 0.0145 & 1.4972 & 0.0145 & 0.0140 & 0.0151 \\
\hline Gini & 0.5326 & 0.5326 & 0.0071 & 0.0067 & 0.0072 & 0.5328 & 0.0073 & 0.0071 & 0.0074 \\
\hline
\end{tabular}

Our third experiment is again concerned with estimation of the GB2 parameters and the related Gini coefficient, but this time under DGP2, using the GMM estimator in (4.5), with and without the $z_{i}$ treated as unknown parameters. The results appear in Table A3 which follows the same format as Table A2 except that, for the unknown $z_{i}$ case, we report a selection of estimates of the $z_{i}$. Our conclusions are similar to those from DGP1. There is no evidence that the estimators are biased. The three variances are very similar, with the asymptotic variance and the average of its estimates slightly understating the Monte-Carlo estimated variance. Having to estimate the group boundaries reduces efficiency, but not by much. Comparing the results from DGP1 and DGP2, we see that, despite substantial differences between the models 
and between the variances of the GLC ordinates, the "true" variances for the parameters are identical as predicted by Proposition 7.

Table A3 Results from Monte Carlo experiment for DGP2

\begin{tabular}{|c|c||c|c|c|c||c|c|c|c||}
\cline { 2 - 9 } \multicolumn{2}{|c||}{} & \multicolumn{4}{c||}{ Known z } & \multicolumn{4}{c||}{ Unknown z } \\
\hline & $\begin{array}{c}\text { True } \\
\text { Par }\end{array}$ & $\begin{array}{c}\text { Average } \\
\text { Est-Par }\end{array}$ & $\begin{array}{c}\text { True } \\
\text { Var }\end{array}$ & $\begin{array}{c}\text { Average } \\
\text { Est-Var }\end{array}$ & $\begin{array}{c}\text { Variance } \\
\text { Est-Par }\end{array}$ & $\begin{array}{c}\text { Average } \\
\text { Est-Par }\end{array}$ & $\begin{array}{c}\text { True } \\
\text { Var }\end{array}$ & $\begin{array}{c}\text { Average } \\
\text { Est-Var }\end{array}$ & $\begin{array}{c}\text { Variance } \\
\text { Est-Par }\end{array}$ \\
\hline$Z_{1}$ & 10.656 & & & & & 10.654 & 0.0159 & 0.0151 & 0.0159 \\
\hline$Z_{2}$ & 17.430 & & & & & 17.438 & 0.0118 & 0.0121 & 0.0118 \\
\hline$z_{10}$ & 70.139 & & & & & 70.153 & 0.0205 & 0.0208 & 0.0205 \\
\hline$z_{18}$ & 236.70 & & & & & 236.69 & 1.36 & 1.63 & 1.36 \\
\hline$z_{19}$ & 343.56 & & & & & 343.65 & 11.39 & 10.47 & 11.50 \\
\hline$b$ & 100.00 & 100.57 & 32.84 & 34.78 & 35.88 & 100.55 & 34.06 & 36.05 & 37.33 \\
\hline$p$ & 1.0000 & 1.0144 & 0.0122 & 0.0123 & 0.0132 & 1.0140 & 0.0125 & 0.0128 & 0.0136 \\
\hline$q$ & 1.5000 & 1.5276 & 0.0417 & 0.0418 & 0.0460 & 1.5268 & 0.0431 & 0.0434 & 0.0477 \\
\hline$a$ & 1.5000 & 1.4976 & 0.0142 & 0.0136 & 0.0146 & 1.4985 & 0.0145 & 0.0140 & 0.0151 \\
\hline Gini & 0.5326 & 0.5328 & 0.0071 & 0.0068 & 0.0072 & 0.5328 & 0.0073 & 0.0071 & 0.0075 \\
\hline
\end{tabular}

Our final experiment is designed to compare the performance of the minimum distance estimator in (3.17) with the least squares estimator in (3.20). Using data generated from DGP1, and with unknown group bounds, two scenarios were considered. In the first scenario the data were generated from the GB2 distribution as in the previous experiments, and the parameters of the distribution were estimated using the GLC specification in (2.8). In the second scenario 10,000 observations were generated consistently with the SCS-GLC specification in (2.5) and grouped into 20 groups of equal size. ${ }^{3}$ The minimum distance and least squares estimators were used to estimate the SCS-GLS parameters which were set at $\mu=40, \beta_{1}=-2, \beta_{2}=0.8$, and $\beta_{3}=3.4$. The results are presented in Table A4 in the same format as those from the earlier experiments. For the least squares variances, we use the "correct" formula in (3.23). The minimum distance estimator has smaller variances in both cases, with the difference being particularly marked for the GB2 distribution. Also, for least squares applied to the GB2

\footnotetext{
${ }^{3}$ To generate the data according to this GLC, we use the inverse-cdf method and equation (3.14).
} 
distribution we see some relatively large differences between the true variances, the average of the estimated variances, and the variance of the estimated parameters. These differences can be attributed to the heavy tail of the assumed distribution since some of the least squares estimates approach values that yield an infinite second moment. ${ }^{4}$

Table A4 Monte Carlo results, minimum distance vs least squares

\begin{tabular}{|c|c|c|c|c|c|c|c|c|c|}
\hline & & & MD & GB2 & & & LS- & GB2 & \\
\hline & $\begin{array}{c}\text { True } \\
\text { Par }\end{array}$ & $\begin{array}{c}\text { Average } \\
\text { Est-Par }\end{array}$ & $\begin{array}{l}\text { True } \\
\text { Var }\end{array}$ & $\begin{array}{c}\text { Average } \\
\text { Est-Var }\end{array}$ & $\begin{array}{c}\text { Variance } \\
\text { Est-Par }\end{array}$ & $\begin{array}{c}\text { Average } \\
\text { Est-Par }\end{array}$ & $\begin{array}{l}\text { True } \\
\text { Var }\end{array}$ & $\begin{array}{c}\text { Average } \\
\text { Est-Var }\end{array}$ & $\begin{array}{c}\text { Variance } \\
\text { Est-Par }\end{array}$ \\
\hline$b$ & 100.00 & 100.56 & 34.06 & 36.50 & 37.52 & 101.37 & 39.25 & 31.14 & 46.85 \\
\hline$p$ & 1.00 & 1.0155 & 0.0125 & 0.0129 & 0.0136 & 1.0610 & 0.0438 & 0.0353 & 0.0569 \\
\hline$q$ & 1.50 & 1.5290 & 0.0431 & 0.0440 & 0.0479 & 1.5984 & 0.1128 & 0.0756 & 0.1422 \\
\hline$a$ & 1.50 & 1.4972 & 0.0145 & 0.0140 & 0.0151 & 1.4678 & 0.0432 & 0.0254 & 0.0521 \\
\hline Gini & 0.5326 & 0.5328 & 0.0073 & 0.0071 & 0.0074 & 0.5326 & 0.0088 & 0.0070 & 0.0074 \\
\hline & & & MD & SCS & & & & SCS & \\
\hline & $\begin{array}{l}\text { True } \\
\text { Par }\end{array}$ & $\begin{array}{c}\text { Average } \\
\text { Est-Par }\end{array}$ & $\begin{array}{l}\text { True } \\
\text { Var }\end{array}$ & $\begin{array}{c}\text { Average } \\
\text { Est-Var }\end{array}$ & $\begin{array}{l}\text { Variance } \\
\text { Est-Par }\end{array}$ & $\begin{array}{c}\text { Average } \\
\text { Est-Par }\end{array}$ & $\begin{array}{l}\text { True } \\
\text { Var }\end{array}$ & $\begin{array}{c}\text { Average } \\
\text { Est-Var }\end{array}$ & $\begin{array}{l}\text { Variance } \\
\text { Est-Par }\end{array}$ \\
\hline$\mu$ & 40.00 & 39.98 & 0.14 & 0.15 & 0.14 & 39.98 & 0.14 & 0.15 & 0.14 \\
\hline$\beta_{1}$ & -2.00 & -2.0924 & 0.2581 & 0.2977 & 0.3065 & -2.0922 & 0.3062 & 0.3384 & 0.3677 \\
\hline$\beta_{2}$ & 0.800 & 0.8014 & 0.0006 & 0.0006 & 0.0006 & 0.8010 & 0.0007 & 0.0007 & 0.0007 \\
\hline$\beta_{3}$ & 3.40 & 3.4922 & 0.2533 & 0.2917 & 0.3014 & 3.4921 & 0.2983 & 0.3297 & 0.3594 \\
\hline Gini & 0.4201 & 0.4199 & 0.0035 & 0.0036 & 0.0035 & 0.4199 & 0.0035 & 0.0036 & 0.0035 \\
\hline
\end{tabular}

\footnotetext{
${ }^{4}$ In fact we have discarded 15 of the Monte Carlo draws in reporting the results for LS-GB2 because of their unreasonably large variances.
} 